\title{
Determination of single and double helical structures in a swirling jet by spectral proper orthogonal decomposition
}

\author{
Yang Zhang (张阳), Maarten Vanierschota \\ KU Leuven, Department of Mechanical Engineering, Celestijnenlaan 300, \\ B-3001 Heverlee, Belgium
}

(Dated: 14 January 2021)

This paper studies the coherent structures found in an annular swirling jet flow undergoing vortex breakdown with control parameters the Reynolds number $R e=8500$ and the swirl number $S w=0.38$. The flow field is simulated using the large eddy simulation (LES) method with a dynamic $k$ model. The first- and second-order statistics of the velocity fields are compared to tomographic particle image velocimetry measurements of the same flow configuration to validate the numerical simulation. The fast Fourier transform of the sampled velocity and pressure signals indicates a precessing vortex core with a frequency of $22 \mathrm{~Hz}$. This frequency is in line with the one detected by spectral proper orthogonal decomposition (SPOD), which is utilized to identify the coherent structures in the near-field region of the swirling flow in the present work. In detail, apart from the single helical structure usually found in swirling flows, a double helix, rarely observed in turbulent swirling jets, is also identified. This structure is not a second-order harmonic mode of the single one as shown by statistical analysis of the mode temporal coefficients. Moreover, the calculation of energy production shows that this coherent precessing motion extracts energy from the mean flow field in the wake behind the bluff-body and in the break up region of the vortex.

a)Electronic mail: maarten.vanierschot@kuleuven.be 


\section{INTRODUCTION}

Swirling flows appear frequently both in nature and in technical applications over a wide range of length scales ${ }^{1 / 3}$. For its many distinctive features including mixing, separation, and stabilization, swirling flows are applied in different types of man-made machines, for instance in cyclone separators and heat exchangers in non-reacting cases, and in industrial furnaces and gas turbines in reacting cases. Owing to its application in swirl burners, there are many investigations about swirling jets, where swirl is served as an approach to control combustion ${ }^{4}$. Introducing swirl into turbulent jets leads to an increase in jet growth, rate of entrainment and rate of decay of the jet. The annular jet flow, i.e. fluid flow through a coaxial cylindrical passage, is extensively used in bluff-body combustors 5 t7 . On the one hand, the wake behind the central body can enhance the fuel-air mixing rate, while on the other hand, the central bluff-body can serve as a fuel injector using cross-flow or co-flow injection $^{8-10}$. In addition to the jet's outer shear layer, the existence of the central bluff-body also introduces an inner shear layer between the jet and the wake, which is characterized by strong anisotropic turbulence $e^{11 \mid 12}$.

Large-scale coherent structures, such as vortex breakdown, which was firstly reported by Peckham and Atkinson $\frac{13}{13}$ on flow over a delta wing, or the accompanying precessing vortex $\operatorname{core}^{14}(\mathrm{PVC})$, are prevalent characteristics in swirling flows. Vortex breakdown occurs when the swirl intensity exceeds a certain threshold and is referred to as an abrupt change in the structure of a vortex core ${ }^{15}$. Different forms of vortex breakdown have been reported, and all of them have several common features, such as an adverse pressure gradient along the streamwise direction, a stagnation point followed by a highly turbulent region of reverse flow further downstream, and a jet-like axial velocity profile upstream and a wake-like velocity profile downstream of the breakdown location 16 . The two most common ones among those different types of breakdown are the asymmetrical spiral type and the nearly axisymmetrical bubble type. Spiral breakdown begins with a rapid deceleration of the vortex core leading to flow stagnation, followed by an abrupt kink which lasts for several turns before breaking up into large-scale turbulence. In combustors, this structure can lead to thermo-acoustical instabilities ${ }^{19}$. The bubble breakdown is also characterized by a stagnation point on the vortex axis, and then followed by an axisymmetric recirculation zone. A comprehensive overview of the occurrence of vortex breakdown or related structures such as the PVC can 
be found in the review papers by Lucca-Negro and O'Doherty ${ }^{20}$ and by Syred ${ }^{14}$.

Various techniques have been proposed to detect coherent structures in turbulent flow fields. Among them, the most widely used include the phase averaging method ${ }^{21}$, the energyranked proper orthogonal decomposition $(\mathrm{POD})^{22}$, and the frequency-ranked dynamic mode decomposition $(\mathrm{DMD})^{23}$. An apparent feature of turbulent flow is that coherent motions could occur at different scales with different frequencies or energy contents. The traditional phase averaging method may not account for this as it treats the coherent fluctuations as a whole. The POD approach looks for the most energetic modes to represent coherent structures with high energy content. However, POD modes could depict coherent motions at multiple different temporal scales because it has no temporal constraints on the empirical modes. The DMD technique, on the other hand, is not a suitable decomposition method for coherent structures with variable frequencies or different intermittently occurring structures with the same frequency. The recently introduced spectral proper orthogonal decomposition (SPOD), which can be regarded as a combination of POD and DMD, as it detects a coherent motion not only with its energy content but also with its frequency ${ }^{24}$. Vanierschot and Ogus ${ }^{25}$ used SPOD to identify PVC in an annular swirling jet in the transitional regime. Kadu et al ${ }^{26}$ employed SPOD to elucidate the physically important structures or modes in a swirling coaxial jet and extended the analysis to the transport of passive scalars.

Large eddy simulation (LES) has become a useful tool for the simulation of turbulent flows. It has the potential to simulate high Reynolds number flow with limited sensitivity to model assumptions. A number of numerical studies about turbulent swirling flows have been conducted using LES, both for scientific interests and for engineering applications. McIlwain and Pollard ${ }^{27}$ used LES to investigate the effects of mild swirl on the near field of a round free jet. Lu et al. ${ }^{28}$ studied features like vortex breakdown, shear layer instability, and vortical-acoustic interactions in turbulent swirling flows by means of the LES technique. A series of studies about coherent structures in turbulent annular swirling jets were carried out through LES by Fröhlich and his co-workers ${ }^{829}\left[31\right.$. Malalasekera et al ${ }^{[32}$ adopted LES to predict the formation of vortex breakdown and associated recirculation zones in isothermal turbulent swirling jets. The work conducted by Wang et al ${ }^{33}$ demonstrated the feasibility of using LES to study complex flow fields in operational devices for engineering applications. The recirculation zones, vortex breakdown bubbles, and PVC were successfully simulated in a Sydney burner using LES by Dinesh and Kirkpatrick ${ }^{34}$ and by Yang and Kæ1 ${ }^{35}$. The 
influence of swirl on a confined coannular swirling jet was investigated by Dinesh et al. $\stackrel{36}{[36}$ using the LES approach. Jones et al. ${ }^{37}$ simulated turbulent confined swirling annular flows using LES to study the behavior of the large-scale coherent structures formed by the high levels of swirl. Zhang et al! $\sqrt[38]{ }$ found that the helical structure associated with the PVC is the most energetic dynamic flow structure in the non-reacting flow fields of a Cambridge swirl burner by performing LES. In addition, LES was utilized to asses the capability of Reynolds-averaged Navier-Stokes simulations on predicting helical instabilities in swirling flows 394 .

In this paper, the LES approach together with the SPOD technique are used to analyse the helical coherent structures in an annular swirling jet. Recently, both single and double helical vortical structures have been identified experimentally in the flow with the same configuration by different coherent structure extraction methods, i.e., POD ${ }^{12}$, phase averaging ${ }^{42}$, and $\mathrm{SPOD}^{43}$. The first intention of this study is to demonstrate that LES can simulate the turbulent annular swirling jet flow with high accuracy in comparison with experimental measurements. First, the LES simulations are validated by time-resolved tomographic PIV measurements of the same flow case ${ }^{43}$. Then, SPOD is conducted on the velocity fields to detect the coherent structures and study their dynamics and influence on the flow field. The paper is structured as follows. The mathematical model and computational methods are introduced in Sec. II. Sec. III illustrates the main details of the SPOD approach. In Sec. IV] we validate the simulation results by experimental data. The SPOD analysis of the velocity fields is given in Sec. V. Finally, we summarize our findings and present the conclusions in Sec. VI,

\section{NUMERICAL SETUP}

\section{A. Governing equations and mathematical model}

For LES, a filter function is applied to the equations of motion, decomposing the flow variables into resolved and modeled components. The governing equations are the threedimensional incompressible filtered Navier-Stokes equations for the resolved velocities $\bar{u}_{i}$ and kinematic pressure $\bar{p}$ (pressure divided by the constant density $\rho$ ):

$$
\frac{\partial \bar{u}_{i}}{\partial t}+\frac{\partial\left(\bar{u}_{i} \bar{u}_{j}\right)}{\partial x_{j}}=-\frac{\partial \bar{p}}{\partial x_{i}}+\nu \frac{\partial^{2} \bar{u}_{i}}{\partial x_{j} \partial x_{j}}-\frac{\partial \tau_{i j}}{\partial x_{j}}
$$




$$
\frac{\partial \bar{u}_{i}}{\partial x_{i}}=0
$$

where $t$ is the time, $x_{i}$ are spatial coordinates, and $\nu$ is the kinematic viscosity of the fluid. In the above formulation, $\tau_{i j}$ is known as the subgrid-scale stress tensor:

$$
\tau_{i j}=\overline{u_{i} u_{j}}-\bar{u}_{i} \bar{u}_{j}
$$

which needs to be modeled in the LES approach. In the present work, we adopt the localized dynamic $k$ model introduced by Kim and Menon ${ }^{44445}$. By defining the subgrid-scale kinetic energy $k_{\text {sgs }}$ as:

$$
k_{s g s}=\frac{1}{2}\left(\overline{u_{i} u_{i}}-\bar{u}_{i} \bar{u}_{i}\right)
$$

the transport equation for it can be derived as:

$$
\frac{\partial k_{s g s}}{\partial t}+\frac{\partial\left(\bar{u}_{j} k_{s g s}\right)}{\partial x_{j}}=-\tau_{i j} \frac{\partial \bar{u}_{j}}{\partial x_{i}}-\varepsilon_{s g s}+\frac{\partial}{\partial x_{j}}\left(\nu_{t} \frac{\partial k_{s g s}}{\partial x_{j}}\right) .
$$

Here, the three terms on the right-hand side stand respectively for the production, dissipation, and diffusion of subgrid-scale kinetic energy $k_{s g s}$. The eddy viscosity $\nu_{t}$ in the above expression is modeled by analogy to the mixing-length hypothesis by choosing the length scale $\bar{\Delta}$ and velocity scale $k_{\text {sgs }}^{1 / 2}$ :

$$
\nu_{t}=C_{\tau} \bar{\Delta} k_{s g s}^{1 / 2}
$$

where $\bar{\Delta}=(\Delta x \Delta y \Delta z)^{1 / 3}$ is the filter width proportional to the grid size in the inertial subrange, and $C_{\tau}$ is a dynamic coefficient. The eddy viscosity $\nu_{t}$ must go towards zero at solid walls since there is no turbulence there. In this case, the van Driest damping function 46 based on the law-of-the-wall proposed by Moin and Kim ${ }^{47}$ is used to account for the reduced growth of the length scale in the near wall region. The localized dynamic $k$ model is a linear eddy-viscosity model, which assumes that the anisotropic part of subgrid-scale residual stress $\tau_{i j}$ is proportional to the filtered rate of strain $\bar{S}_{i j}{ }_{48}$ :

$$
\tau_{i j}-\frac{2}{3} k_{s g s} \delta_{i j}=-2 \nu_{t} \bar{S}_{i j}
$$

with

$$
\bar{S}_{i j}=\frac{1}{2}\left(\frac{\partial \bar{u}_{i}}{\partial x_{j}}+\frac{\partial \bar{u}_{j}}{\partial x_{i}}\right)
$$


In addition, supposing that the subfilter dissipation rate $\varepsilon_{s g s}$ only depends on $k_{s g s}$ and $\bar{\Delta}$, the following formula can be acquired by dimensional analysis:

$$
\varepsilon_{s g s}=C_{\varepsilon} \frac{k_{s g s}^{3 / 2}}{\bar{\Delta}} .
$$

Again, $C_{\varepsilon}$ is another model coefficient which is calculated dynamically. The model coefficients $C_{\tau}$ and $C_{\varepsilon}$ can be determined based on the dynamic procedure ${ }^{49}$ by introducing a new test filter with width $\widetilde{\Delta}=2 \bar{\Delta}$, as depicted in detail in Lilly $\sqrt{50}$ and in Kim and Menon 4 .

\section{B. Computational domain and numerical method}
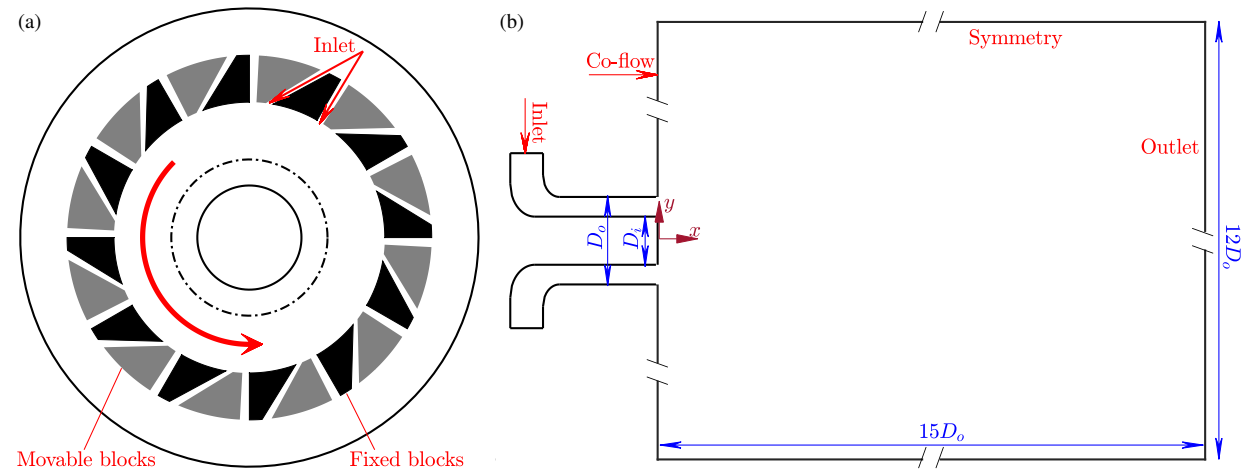

FIG. 1. (a) A schematic layout of the swirl generator. (b) A sketch of the computational flow domain in the $x-y$ plane.

As the swirling velocity profile has a large influence on the structures in the jet, the swirl generating device was also implemented in the model 151 . Two different ways are generally adopted to create swirling flows: the use of guide vanes to divert the axial flow tangentially or the tangential injection of (a part of the) fluid into a main passage. In this work, the second method is adopted and the rotational motion of the jet is induced by a swirler with twelve fixed and movable blocks, in which the fluid can be injected both radially and tangentially. A schematic layout of the swirl generator is shown in figure 1(a). The desired swirl intensity is acquired by rotating the movable blocks to alter the distribution between the tangential and axial flows ${ }^{52}$. Furthermore, the swirl intensity can be estimated in advance based on the geometry of the swirl generator and the opening angles between the fixed/movable blocks 53 , although this estimated value is usually larger than the one obtained from the velocity profiles at the jet orifice ${ }^{5}$. 
The fluid flowing through the swirl generator is guided into an annular pipe whose outer and inner diameters are $D_{o}=27 \mathrm{~mm}$ and $D_{i}=18 \mathrm{~mm}$, respectively. Then, the annular swirling jet issues into the surrounding medium that is at rest in a large cylindrical domain. The cross section of the computational domain in the $x-y$ plane is presented in figure 1(b). The length and diameter of the cylindrical domain are $15 D_{o}$ and $12 D_{o}$, respectively. In addition, figure 1(b) also displays the boundary conditions used in the numerical simulation. The fluid is injected simply with a uniform velocity profile $u_{i n}$ at the inlet and a co-flow is fed in the outer region of the jet with a mild velocity $u_{c}=3 \% u_{i n}$. The no-slip velocity boundary condition is applied at the walls of the inlet duct and the end wall of the central bluff-body. For the velocity at the lateral wall of the unconfined domain and at the outlet, the zero gradient boundary condition is employed. A fixed value boundary condition is imposed for pressure at the far-field boundary, while zero gradient boundary condition is applied to all other boundaries such as inlet, co-flow, and the walls. As for the LES, the turbulent quantity $k_{s g s}$ is set with a fixed value on the inlet, co-flow, and solid walls, and with a zero-gradient condition imposed at the lateral symmetry wall and outlet.

The structured multi-block approach is used to generate computational grids. Also, all the grid cells are hexahedral to meet the requirement for aspect ratio, non-orthogonality, and skewness. Typically, the computational domain describing a $\pi / 6$ angular sector of the swirl generator is shown in figure 2(a). Different views of the computational grids close to the jet orifice are given in figures 2(b) and 2(c). The hexahedral structured grid is clustered in the wall-normal direction to resolve the viscous boundary layer. Besides, the grid size is finer near the jet central region to be able to well capture the large velocity and pressure gradients caused by the vortex breakdown phenomenon. The minimum grid spacing in the axial direction is located at the jet nozzle with $\Delta x=D_{o} / 600$ and the minimum grid spacing in the radial direction is at the wall of the annular channel with $\Delta r=D_{o} / 1200$. The number of elements is about 10.5 million in total and there are 384 cells in the azimuthal direction. The open source computational fluid dynamics code OpenFOAM ${ }^{\sqrt{54}}$ is utilized to simulate the annular swirling jet in this study. The coupled pressure/velocity fields are obtained using the PISO (pressure implicit with splitting of operators) algorithm ${ }^{55}$ at each time step with the geometric-algebraic multi-grid (GAMG) approach to solve the corrected pressure equation. The temporal derivative term is discretized using a second-order implicit Euler scheme. The spatial discretizations are second-order upwind for the convective terms and 

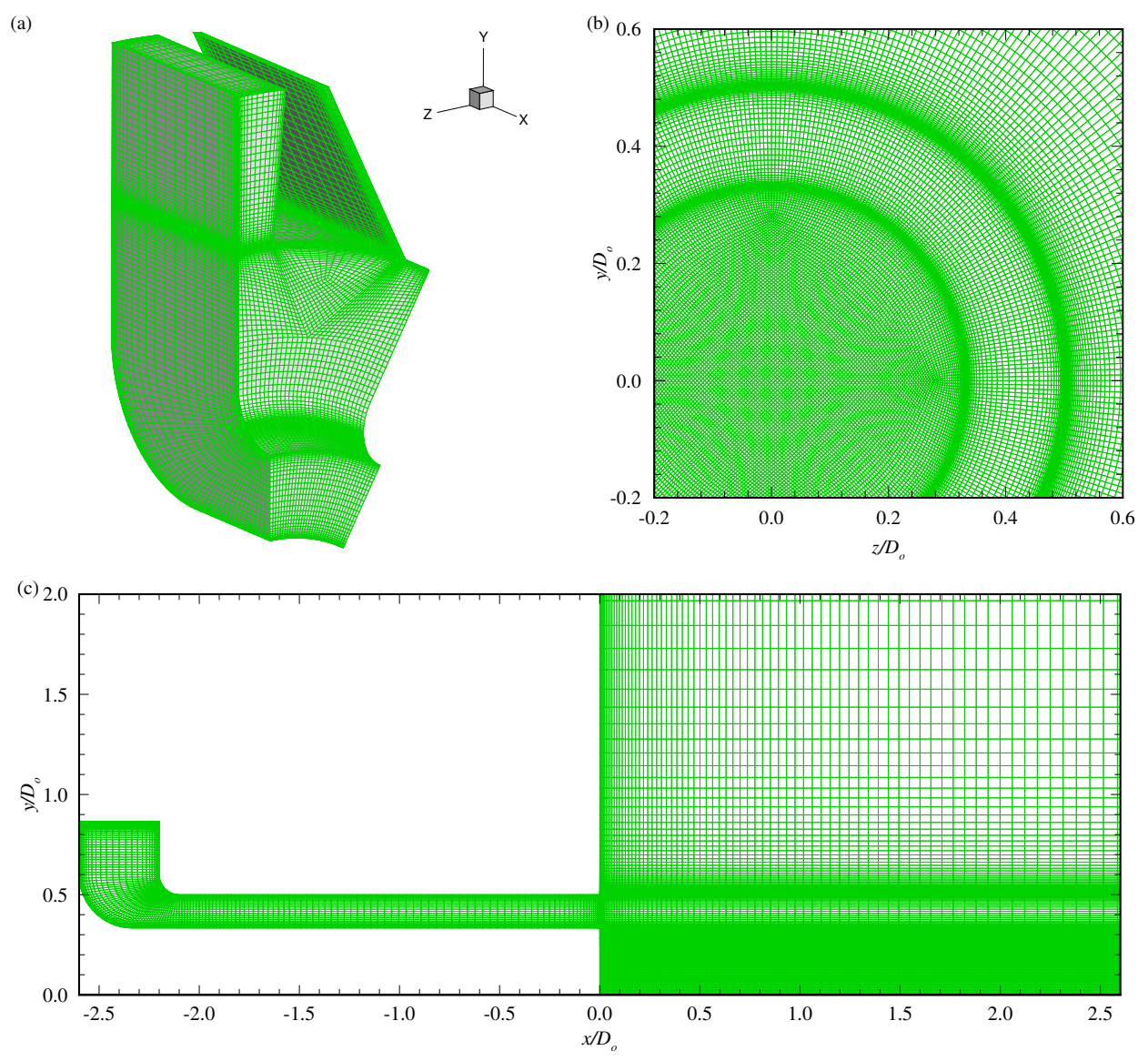

FIG. 2. Computational grid used in the numerical simulation: (a) a section of the mesh in the swirl generator, (b) axisymmetric plane perpendicular to the jet axis at $x / D_{o}=0$, and (c) cross-section in the $z / D_{o}=0$ plane.

a central difference scheme for the diffusive terms, respectively. The linear interpolation scheme is used to calculate flow variables such as the volumetric flux on the control surface. The $y^{+}$value is 1.43 on average calculated from the near-wall mesh and its maximum is about 4.56 close to the inlet. A fixed time step $\Delta t=5 \times 10^{-6} \mathrm{~s}$ is chosen to fulfill the Courant-Friedrichs-Lewy (CFL) condition and the CFL number does not exceed 0.56 during the simulation.

To estimate the resolution of the LES data in the current study, we take the measure proposed by Pope ${ }^{56}$, who suggested that $80 \%$ of the turbulent kinetic energy should be resolved in LES. The profiles of resolved turbulent kinetic energy $k_{\text {res }}=\frac{1}{2}\left\langle\bar{u}_{i}^{\prime} \bar{u}_{i}^{\prime}\right\rangle$ and total turbulent kinetic energy $k_{t o t}=k_{\text {res }}+\left\langle k_{\text {sgs }}\right\rangle$ at four different axial locations immediately downstream of the central bluff-body are shown in figure $3(\mathrm{a})$. Here, the notation $\langle\cdot\rangle$ and 
' denote the time average and fluctuation of the variables, respectively. It is seen that the turbulent kinetic energy is larger close to the inner and outer shear layers, and the CRZ regions at $x / D_{o}=0.2$ whereas it is greater near the vortex breakdown bubble further downstream. This is in line with former experimental studies 12 . The ratio between $k_{\text {res }}$ and $k_{t o t}$ at those four axial positions is shown in figure $3(\mathrm{~b})$, which demonstrates that in general more than $90 \%$ of the turbulent kinetic energy is resolved in the near field of the annular jet. In addition, the grid resolution ensures that at least $80 \%$ of the turbulent kinetic energy is resolved in the whole flow domain (not shown here).
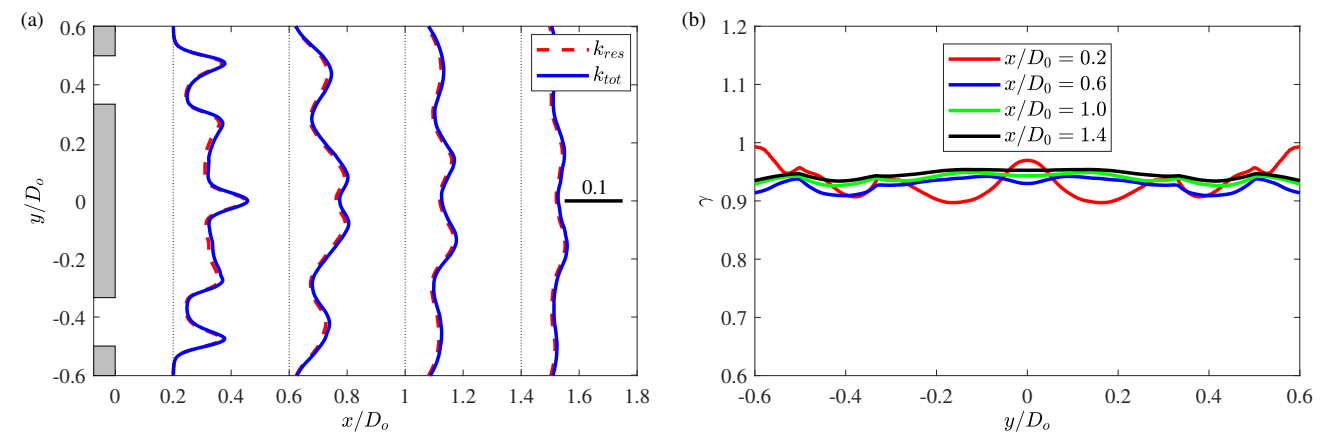

FIG. 3. Spatial resolution estimation of the LES case: (a) the profiles of resolved $k_{r e s}$ and total $k_{t o t}$ turbulent kinetic energy at four different axial locations $\left(x / D_{o}=0.2,0.6,1.0,1.4\right)$, (b) the ratio $\gamma$ between $k_{\text {res }}$ and $k_{t o t}$.

\section{COHERENT STRUCTURE EXTRACTION METHOD}

In research on turbulence, the Reynolds decomposition is widely used to extract the stochastic fluctuation of a specific physical quantity. However, this approach is not suitable for turbulent flow sustaining a well-organised large-scale coherent motion, for instance, the annular swirling jet flow concerned in the present work. Under this condition, the employment of Reynolds decomposition would overestimate the (turbulent) stochastic part of the flow as the coherent structure is a source of fluctuation as well. Nevertheless, this deficiency can be overcome by the triple decomposition introduced by Hussain and Reynolds $\frac{57}{\text {, con- }}$ sidering that there is a periodic motion in the flow field. This method decomposes a given variable such as the velocity vector $\boldsymbol{u}(\boldsymbol{x}, t)=(u, v, w)$ into three different components:

$$
\boldsymbol{u}(\boldsymbol{x}, t)=\langle\boldsymbol{u}(\boldsymbol{x}, t)\rangle+\boldsymbol{u}^{\dagger}(\boldsymbol{x}, t)+\boldsymbol{u}^{\ddagger}(\boldsymbol{x}, t) .
$$


Here, $\langle\boldsymbol{u}(\boldsymbol{x}, t)\rangle$ is the time-averaged value, $\boldsymbol{u}^{\dagger}(\boldsymbol{x}, t)$ is the phase-averaged or coherent part, and $\boldsymbol{u}^{\ddagger}(\boldsymbol{x}, t)$ is the (turbulent) stochastic motion. This triple decomposition approach forms a basis for various subsequent techniques such as the phase averaging method, POD and DMD.

In terms of the SPOD technique, its formulation is quite similar to the classical snapshot $\mathrm{POD}^{24}$. Taking $N$ time series data collected from $M$ grid points in a three-dimensional volume with $N \ll M$ into account, the fluctuating part in equation 10 is split into a collection of spatial modes:

$$
\boldsymbol{u}(\boldsymbol{x}, t)=\langle\boldsymbol{u}(\boldsymbol{x}, t)\rangle+\boldsymbol{u}^{\S}(\boldsymbol{x}, t)=\langle\boldsymbol{u}(\boldsymbol{x}, t)\rangle+\sum_{i=1}^{N} a_{i}(t) \boldsymbol{\Phi}_{i}(\boldsymbol{x}),
$$

with the fluctuation $\boldsymbol{u}^{\S}(\boldsymbol{x}, t)$ given by:

$$
\boldsymbol{u}^{\S}(\boldsymbol{x}, t)=\boldsymbol{u}^{\dagger}(\boldsymbol{x}, t)+\boldsymbol{u}^{\ddagger}(\boldsymbol{x}, t)
$$

Here, $\boldsymbol{\Phi}_{i}(\boldsymbol{x})$ is the SPOD mode and $a_{i}(t)$ is the corresponding mode temporal coefficient. The difference between POD and SPOD is that the correlation matrix $\boldsymbol{R}$ (with a size of $N \times N)$ in POD

$$
R_{i, j}=\frac{1}{N}\left[\boldsymbol{u}^{\S}\left(\boldsymbol{x}, t_{i}\right)\right]^{T}\left[\boldsymbol{u}^{\S}\left(\boldsymbol{x}, t_{j}\right)\right]
$$

is filtered in SPOD by a filter coefficients vector $\boldsymbol{g}$ with a length of $\left(2 N_{f}+1\right)$ to get the filtered correlation matrix $\boldsymbol{S}$ :

$$
S_{i, j}=\sum_{k=-N_{f}}^{N_{f}} g_{k} R_{i+k, j+k} .
$$

The temporal coefficient $\boldsymbol{a}_{i}=\left[a_{i}\left(t_{1}\right), a_{i}\left(t_{2}\right), \ldots, a_{i}\left(t_{N}\right)\right]^{T}$ and mode energies $\lambda_{i}$ are obtained from the eigenvectors and eigenvalues of matrix $\boldsymbol{S}$ :

$$
\boldsymbol{S} \boldsymbol{a}_{i}=\lambda_{i} \boldsymbol{a}_{i}, \quad \lambda_{1} \geqslant \lambda_{2} \geqslant \cdots \geqslant \lambda_{n} \geqslant 0
$$

Then, the spatial modes $\boldsymbol{\Phi}_{i}(\boldsymbol{x})$ are calculated by projection of the snapshots onto the temporal coefficients:

$$
\boldsymbol{\Phi}_{i}(\boldsymbol{x})=\frac{1}{N \lambda_{i}} \sum_{j=1}^{N} a_{i}\left(t_{j}\right) \boldsymbol{u}^{\S}\left(\boldsymbol{x}, t_{j}\right) .
$$

Moreover, to identify linked SPOD modes (modes with similar spectral content of the temporal coefficients), DMD is applied to the SPOD coefficients $\boldsymbol{a}=\left[\boldsymbol{a}_{1}, \boldsymbol{a}_{2}, \ldots, \boldsymbol{a}_{N}\right]$ since the 
modes from a pair describing a single structure have the same spectral content $\stackrel{24}{ }$. Finally, the large-scale coherent part $\boldsymbol{u}^{\dagger}(\boldsymbol{x}, t)$ can be reconstructed by the linked SPOD modes:

$$
\boldsymbol{u}^{\dagger}(\boldsymbol{x}, t)=\sum_{r}\left[a_{p}^{r}(t) \boldsymbol{\Phi}_{p}^{r}(\boldsymbol{x})+a_{q}^{r}(t) \boldsymbol{\Phi}_{q}^{r}(\boldsymbol{x})\right]
$$

where a mode pair $r$ consists of SPOD modes with indices $p$ and $q$. In this work, the SPOD is conducted on the filtered velocity field $\overline{\boldsymbol{u}}(\boldsymbol{x}, t)=(\bar{u}, \bar{v}, \bar{w})$ from the LES.

\section{GLOBAL FEATURES}

\section{A. Control parameters}

The control parameters of the system, i.e., the Reynolds number $R e$ and swirl number $S w$ are calculated based on the velocity profiles at the nozzle exit. The Reynolds number is defined as

$$
R e=\frac{u_{0} D_{h}}{\nu}
$$

where $u_{0}=0.95 \mathrm{~m} / \mathrm{s}$ is the mean axial velocity in the annular channel, $D_{h}=9 \mathrm{~mm}$ is the hydraulic diameter of the annular pipe, and $\nu=1.005 \times 10^{-6} \mathrm{~m}^{2} / \mathrm{s}$ is the kinematic viscosity of the working fluid. The swirl number, which measures the ratio between the axial flux of tangential momentum and the axial flux of axial momentum divided by a characteristic radius, is given by

$$
S w=\frac{\int_{\frac{D_{i}}{2}}^{\frac{D_{o}}{2}} 2 \pi \rho\langle\bar{u}\rangle\langle\bar{w}\rangle r^{2} d r}{\frac{D_{o}}{2} \int_{\frac{D_{i}}{2}}^{\frac{D_{o}}{2}} 2 \pi \rho\langle\bar{u}\rangle^{2} r d r},
$$

where $\langle\bar{u}\rangle$ and $\langle\bar{w}\rangle$ are the mean axial and tangential velocity measured at $x / D_{o}=0$, respectively. The computed Reynolds number $R e$ is around 8500, and the swirl number $S w$ is calculated as 0.38 . The Reynolds number is the same as the one used in the experimental study for validation. However, it is rather low to be able to have sufficient temporal resolution

of the time-resolved PIV measurements as at higher Reynolds numbers the frequencies of precession would also increase 43 . 

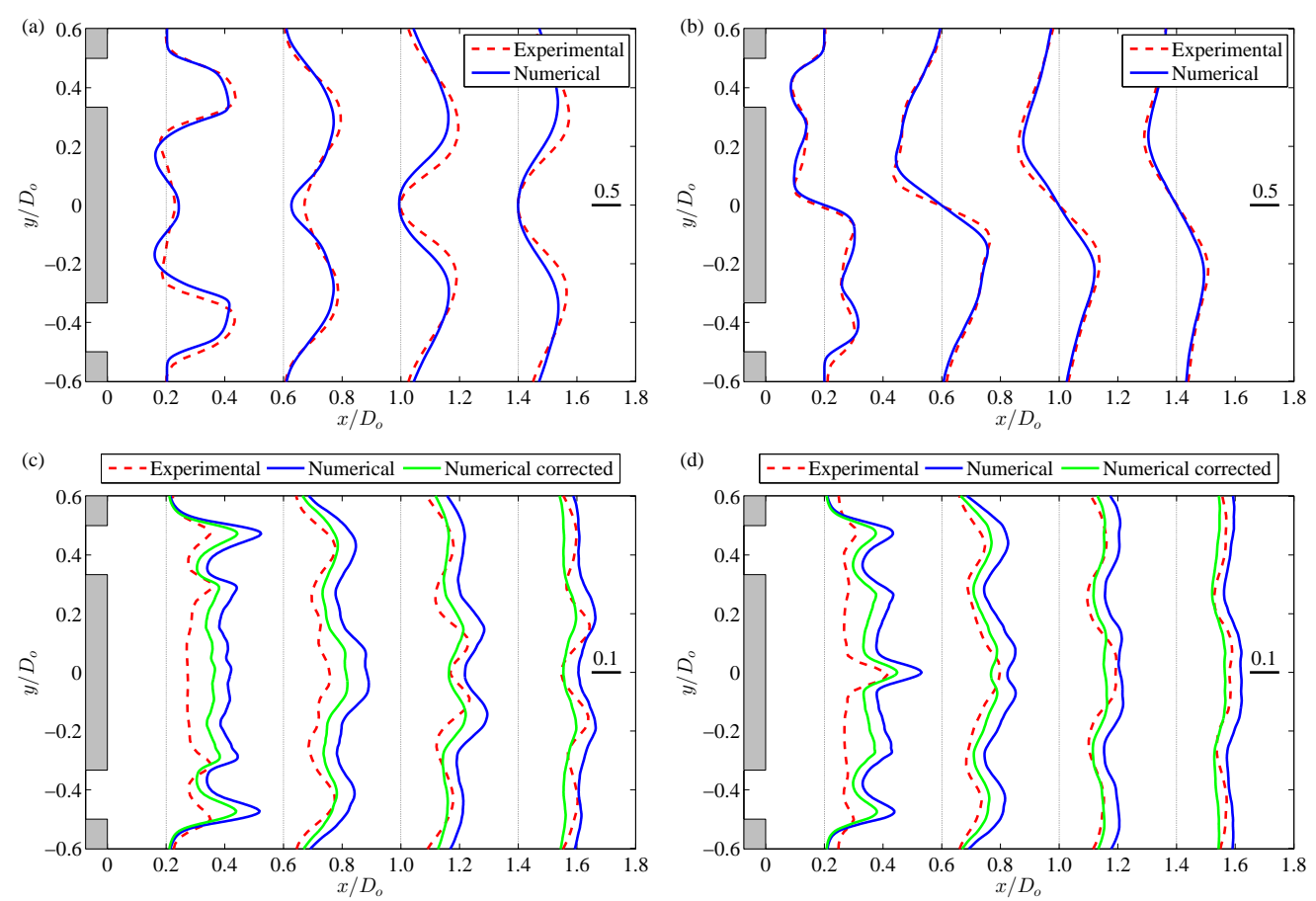

FIG. 4. Radial distributions of time-averaged axial/azimuthal velocities and root-mean-square values of axial/azimuthal velocity fluctuations normalized by $u_{0}$ for the annular swirling jet at different axial positions $\left(x / D_{o}=0.2,0.6,1.0\right.$ and 1.4) in a central $x-y$ plane: (a) time-averaged axial velocity $\langle\bar{u}\rangle / u_{0}$, (b) time-averaged azimuthal velocity $\langle\bar{w}\rangle / u_{0}$, (c) root-mean-square values of streamwise velocity fluctuation $\bar{u}_{r m s} / u_{0}$, (d) root-mean-square values of azimuthal velocity fluctuation $\bar{w}_{r m s} / u_{0}$.

\section{B. Experimental validation}

To validate the LES, the first- and second-order statistics of the numerical results are compared with experimental data obtained by tomographic particle image velocimetry 12 . Figures 4(a) and 4(b) depict the normalized time-averaged streamwise and tangential velocity profiles at four different axial locations $\left(x / D_{o}=0.2,0.6,1.0,1.4\right)$ in an $x$ - $y$ plane, respectively. The solid lines illustrate the numerical data and the dashed lines correspond to the experimental measurements. Similar to other observations in experimental studies ${ }^{11}$, the axial velocity shows a wake-like profile downstream of the annular jet nozzle. The LES velocity profiles in the immediate near field agree very well with the measured profiles (note that the experimental velocity profiles are a little asymmetric). However, the numerical velocity profiles in the jet central region around $x / D_{o}=0.6$ are underestimated, which 
means that the stagnation point caused by vortex breakdown moves a little upstream (the location of the stagnation point is $x / D_{o} \approx 0.8$ for the numerical simulations and 0.9 for the experiments). Further downstream, the axial velocity magnitude of the LES data is smaller (for instance at $x / D_{o}=1.4$ ) because the size of the predicted vortex breakdown bubble is larger when compared to the experimental one. Most probably this is related to small differences in swirl number as the size of the breakdown bubble is extremely sensitive to the swirl number around the critical regime ${ }^{11}$. The profiles of the rms (root mean square) values of streamwise and azimuthal velocity fluctuations are given in figures $4(\mathrm{c})$ and $4(\mathrm{~d})$. It is seen that the LES results show larger magnitudes as compared with the experimental measurements. Besides, the difference between the rms velocity profiles is larger than the time-averaged velocity profiles. This discrepancy is caused by the spatial resolution of the tomographic PIV measurements. Studies on similar flow topologies have shown that LES can predict the fluctuations well, but those studies compared LES with LDA data and LDA

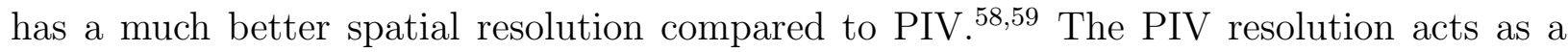
spatial filter, reducing the amplitude of the fluctuations 11 . The maximal wavelength which can be captured is equal to $\pi / \Delta$, where $\Delta$ is the size of the interrogation area $(1.12 \mathrm{~mm}$ in the study of Percin et al $\left.{ }^{[12}\right)$. This spatial averaging induces large errors in the estimation of the turbulent dissipation rate and the second order moments of the velocity field. Table I shows the Kolmogorov length scale $\eta$, the dissipation rate $\epsilon$ and the integral length scale $L$ computed based on the experimental data of Percin et al ${ }^{[12}$ and on data from the current LES study. As can be seen, due to the spatial averaging, the dissipation rate is underestimated by a factor 3, the integral length scale overpredicted by a factor 2 and the Kolmogorov length scale overestimated by $33 \%$ in the experiments compared to the simulations. Based on the study of Alekseenko et al. $\underline{60}$, the underprediction factor $f$ of the second order moments for the ratio $\Delta / L=0.8$ can be up to $60 \%$ in certain regions of the flow with high gradients. 60 which is much larger than the $8 \%$ estimated from the experimental data of Percin et al.12. Therefore, exact values of the rms values can not be compared between experiments and LES, especially near the nozzle. If one scales the LES results with the underprediction factor (green lines in figures 4(c) and 4(d)) the shapes of the profiles are very similar at different axial locations. Further downstream, the profiles are overcorrected as the correction factor $f$ is strongly dependent on the location in the flow and decreases downstream. As such, LES can well capture the dynamics of the swirling flow. The overprediction of the bubble size 
TABLE I. Maximum dissipation rate $(\epsilon)$, Kolmogorov $(\eta)$ and integral $(L)$ length scales of the flow compared to the interrogation area size $\Delta$ in the study of Percin et al.$^{12}$

\begin{tabular}{|l|l|l|l|l|l|l|}
\hline & $\Delta[\mathrm{mm}]$ & $\eta[\mathrm{mm}]$ & $\epsilon\left[\mathrm{m}^{2} / \mathrm{s}^{3}\right]$ & $L[\mathrm{~mm}]$ & $\Delta / \eta[-]$ & $\Delta / L[-]$ \\
\hline Experimenta1 & 1.12 & 0.024 & 3.13 & 2.7 & 46 & 0.42 \\
\hline LES & & 0.018 & 9.5 & 1.4 & 62 & 0.8 \\
\hline
\end{tabular}

by LES causes also a difference in rms velocity profiles between numerical and experimental data near $x / D_{o}=1.4$. The cause for this is that the PVC is located on the boundary of the breakdown bubble and its motion leads to strong turbulent mixing.

\section{Mean velocity fields}

Figures 5(a) and 5(b) plot the distribution of the normalized time-averaged streamwise and azimuthal velocity in the $z / D_{o}=0$ plane, respectively. According to figure $5(\mathrm{a})$, there are two dominant regions of backflow in the near field of the annular swirling jet. The first one comes from the flow separation behind the central bluff-body, and it is toroidal in shape due to the centrifugal force introduced by swirl close to the jet orifice. Usually, the generation of this recirculation region is to provide a long enough flow residence time for fuel and oxygen to mix and combust in industrial swirl burners ${ }^{5 \mid 14}$. Further downstream around $x / D_{o}>0.8$, there is another recirculation region that is caused by vortex breakdown. Experimental studies have shown that the vortex breakdown bubble would move upstream
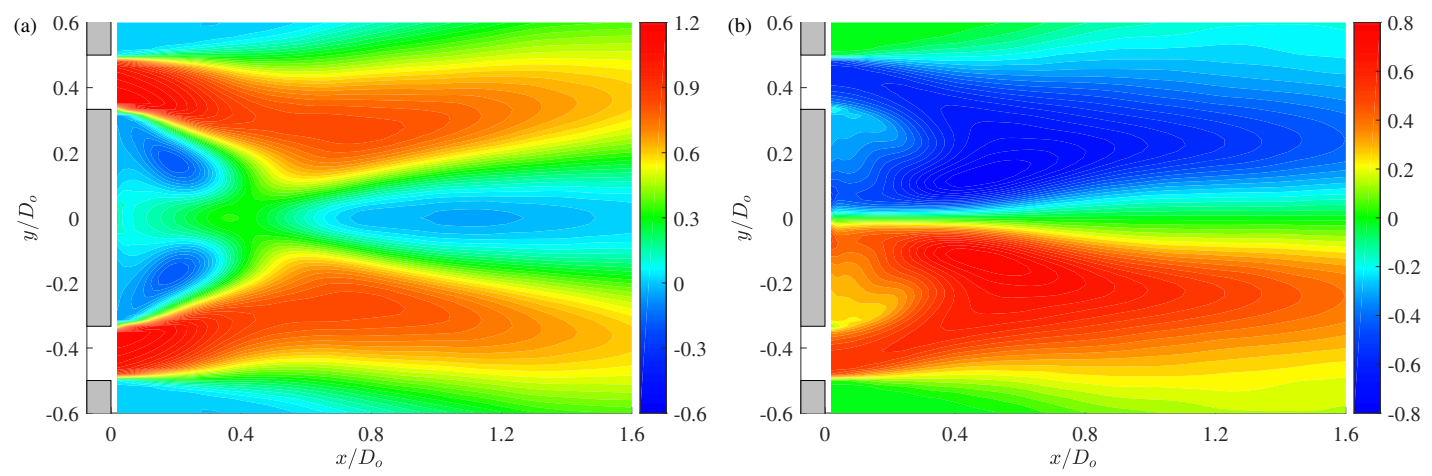

FIG. 5. Distribution of time-averaged velocity fields normalized by $u_{0}$ in the $z / D_{o}=0$ plane: (a) mean axial velocity $\langle\bar{u}\rangle / u_{0}$, (b) mean azimuthal velocity $\langle\bar{w}\rangle / u_{0}$. 
(a)

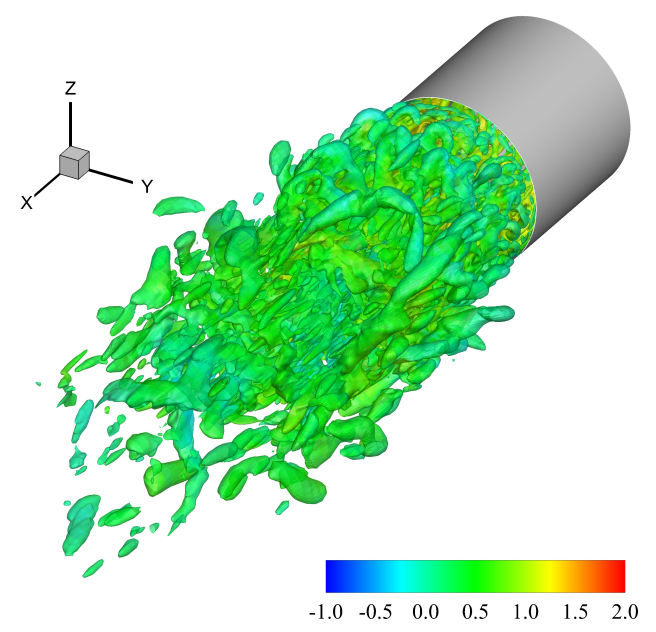

(b)
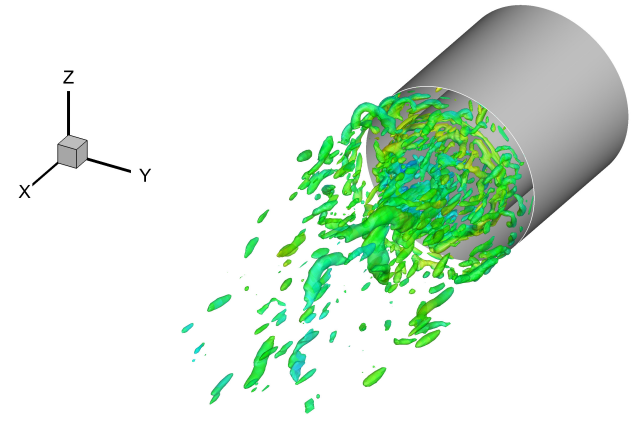

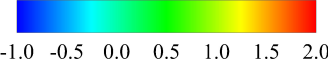

FIG. 6. Vortical structures shown by iso-surface of positive $Q$ : (a) $Q=10^{4} 1 / \mathrm{s}^{2}$, (b) $Q=10^{5}$ $1 / \mathrm{s}^{2}$. The iso-surface is colored by the streamwise velocity $\bar{u}$ (unit $\mathrm{m} / \mathrm{s}$ ).

towards the toroidal recirculation zone, and they will be merged together as the swirl strength increases ${ }^{1161}$. From figure 5(b) we can observe that angular momentum still exists in these recirculation regions, but the magnitude of tangential velocity is much smaller due to flow stagnation.

\section{Instantaneous flow structures}

The instantaneous flow structures are shown in figure 6. This figure shows vortical structures based on the Q-criterionn ${ }^{62}$. Here, $Q=\frac{1}{2}\left(\|\bar{W}\|^{2}-\|\bar{S}\|^{2}\right)$, where $\bar{W}_{i j}=\frac{1}{2}\left(\frac{\partial \bar{u}_{i}}{\partial x_{j}}-\frac{\partial \bar{u}_{j}}{\partial x_{i}}\right)$ is the vorticity tensor. The $Q$-criterion is a region-based method, which identifies the vortex core by a scalar threshold. In addition, the size of the extracted vortex core depends on the selected threshold. By defining two thresholds, figure 6 shows that the vortices in the inner shear layer (which are convected back to the nozzle, i.e. a negative axial velocity) are generally stronger compared to the vortices in the outer shear layer (which are convected away from the nozzle, i.e. a positive axial velocity). At the center near the nozzle, a large vortex can be identified as a double helical structure, as shown in figure 6(b). In order to better identify this helical structure, isocontours of pressure are shown in figure 7 as the pressure in a vortex core is low, isocontours of pressure can help identifying them. In this 
figure, two arbitrary time instants were chosen to show the intermittent presence of both a double helix (figures 7(a) and (b)) and a single helix (figures 7(d) and (e)). The axial vorticity component in figures 7 (c) and 7 (f) shows the presence of multiple small scale vortices rotating in the same direction as the large scale one. A detail of these small scale structures in the inner shear layer is shown in figure 8. Three different arbitrary time instants are chosen where (1) no large scale structure (left), (2) the single helix (middle) and (3) the double helix (right) are present. Near the nozzle, these small scale vortices originate from shear layer instabilities and their main direction is in the azimuth. They are convected downstream along helical paths and tilted by the main flow towards the axial flow direction. Afterwards, the longitudinal vortices are convected downstream towards the outlet.
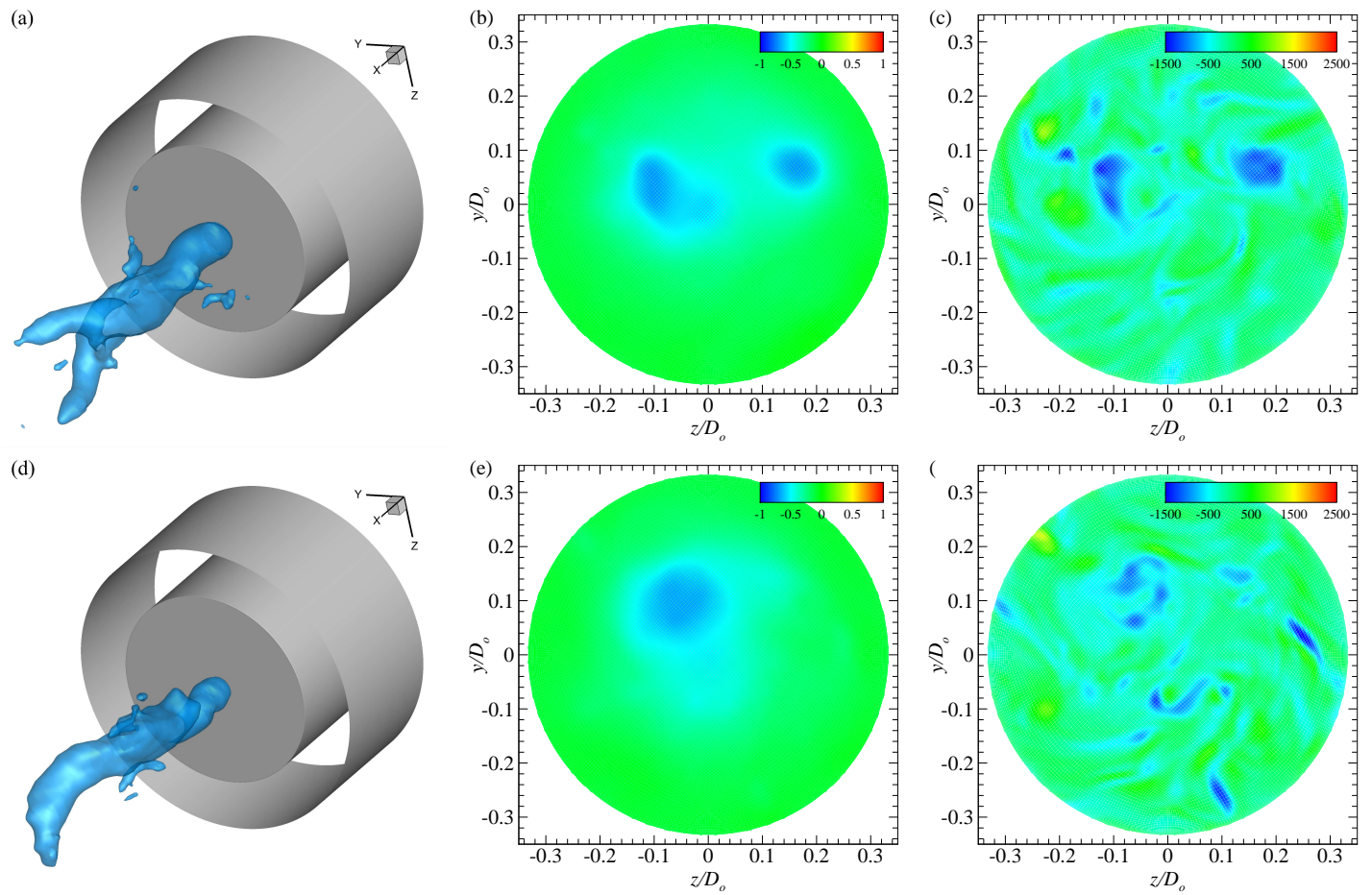

FIG. 7. Double (a) and single (d) helical structures obtained form the instantaneous pressure field by an iso-surface of $-0.54 \mathrm{~m}^{2} / \mathrm{s}^{2}$ at two arbitrary time instants. The slices of corresponding pressure (in (b) and (e), unit $\mathrm{m}^{2} / \mathrm{s}^{2}$ ) and vorticity component $\omega_{x}=\frac{\partial \bar{w}}{\partial y}-\frac{\partial \bar{v}}{\partial z}$ (in (c) and (f), unit $1 / \mathrm{s}$ ) at $x / D_{o}=0.9$ are shown as well. 

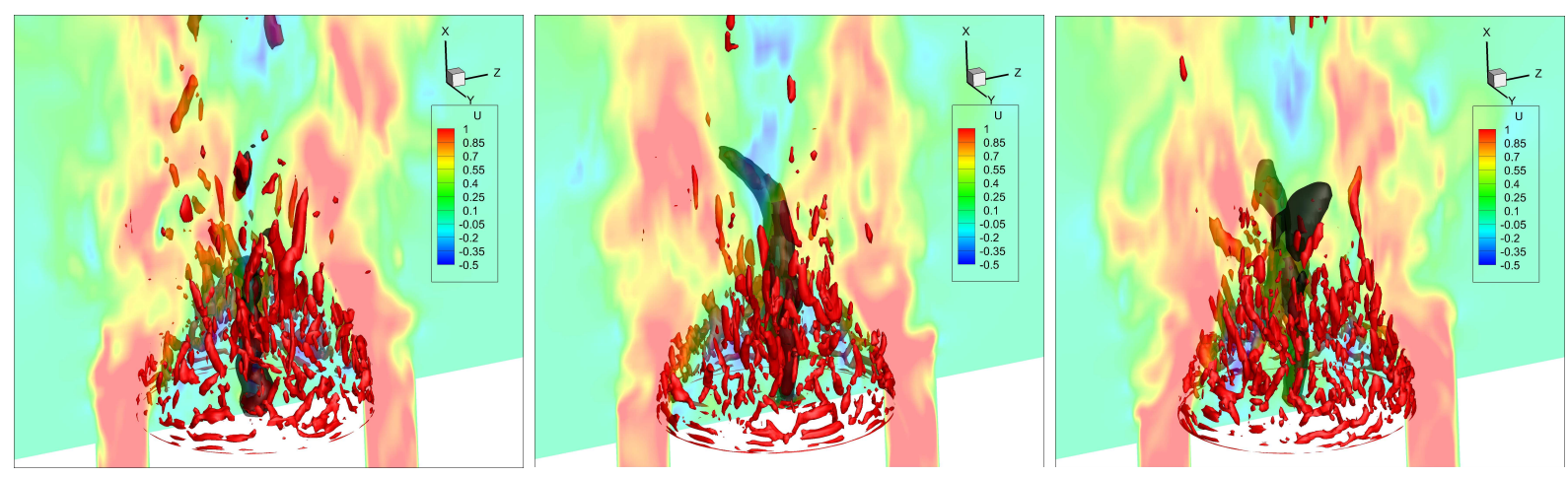

FIG. 8. Close-up on the vortical structures near the nozzle in the inner shear layer. The vortices are identified by the red isosurfaces of $Q=1000001 / \mathrm{s}^{2}$. The large scale helical structures are identified by black isosurfaces of $\bar{p}=-0.65 \mathrm{~m}^{2} / \mathrm{s}^{2}$. The slice shows contours of the axial velocity.

\section{IMPLEMENTATION OF SPECTRAL PROPER ORTHOGONAL DECOMPOSITION}

\section{A. Spectral analysis}

The occurrence of coherent structures is usually indicated by oscillations in the velocity and pressure values at various spatial locations in the flow field. An analysis of their spectral information is applied to obtain the frequency of rotation of large scale structures. In the simulation, time signals of velocity components and pressure are recorded every time step at several different radial positions and on the jet axis. In particular, the monitored velocity variables and pressure at the stagnation point during two seconds are plotted in figures 9(a) and 9(c), respectively. The signals chosen in this location are closely related to the precessing vortex core since the stagnation point is induced by the vortex breakdown phenomenon. As depicted in the figures, the velocity and pressure series clearly show turbulent behavior, i.e. they fluctuate intensively in the time domain around the mean value indicated by the dashed line in the figures. Figures 9(b) and 9(d) illustrate the corresponding power spectral density of the velocity and pressure fluctuations using Welch's method as shown in figures 9 (a) and 9(c). A distinct peak indicating the presence of a coherent structure at frequency $f \approx 22 \mathrm{~Hz}$ can be observed in these figures, which is labeled by the dashed lines and the arrow. This frequency can also be revealed by the SPOD analysis as will be shown later. Recently, a coherent structure in an annular swirling jet with a precessing frequency 

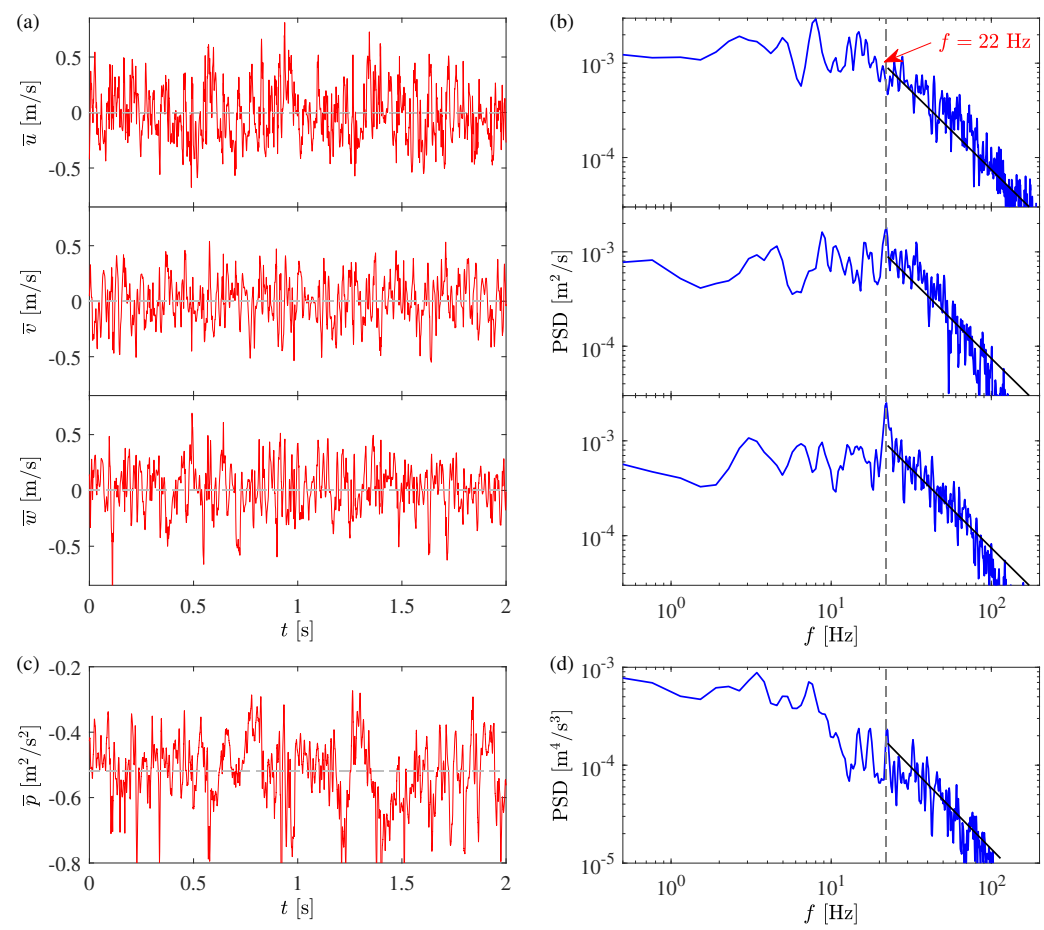

FIG. 9. The sampled velocity (a) and pressure (c) signals at the stagnation point during 2 seconds, the corresponding power spectral density distribution of velocity (b) and pressure (d) fluctuations. The arrow indicates the main peak of the PSD distributions with a frequency of around $22 \mathrm{~Hz}$. The slopes represent the $-5 / 3$ power law of Kolmogorov.

of $28 \mathrm{~Hz}$ has been reported experimentally by POD analysis of the pressure fields ${ }^{12}$, and by phase averaging and SPOD of the velocity fields ${ }^{42 / 43}$. In comparison with the experimental results, the numerical data show a frequency shift about the precessing frequency. This difference can be attribute to the discrepancy of the azimuthal velocity profiles upstream of the vortex breakdown bubble as plotted in figure 4(b). The investigations in isothermal swirling flow have shown that the frequency of precessing vortex core is proportional to the tangential velocity gradient close to the jet central axis $\frac{14 \mid 20}{2}$, which is under predicted by the simulation. Moreover, the slopes in figures 9(b) and 9(d) represent the Kolmogorov's $-5 / 3$ power law, which implies that the grid resolution in the simulation is fine enough to predict the characteristics in the inertial subrange reasonably well. 


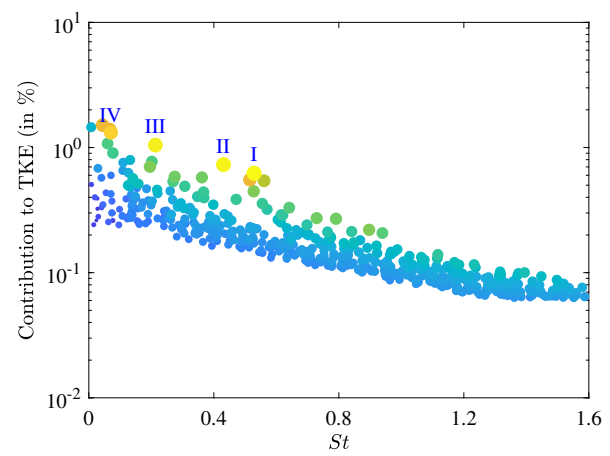

FIG. 10. The identified SPOD mode pairs and their percentage energy content. The Roman numerals mark the first four mode pairs with the highest magnitude of correlation.

\section{B. Spectral proper orthogonal decomposition modes}

To conduct the SPOD on the instantaneous three-dimensional flow fields, the velocity fields are sampled at a fixed time interval during the numerical simulation. In this study, the time interval is chosen to be $2.5 \mathrm{~ms}$ between two snapshots and a total of $N=1200$ snapshots are recorded. The extracted SPOD mode pairs and their energy content are displayed in figure 10. In the figure, each dot represents one mode pair and the size of it indicates the magnitude of the correlation between two linked harmonic modes. Furthermore, the first four ranked mode pairs with the highest harmonic correlation are labeled with Roman numerals. Here, the dimensionless Strouhal number $S t$ is defined as $S t=f D_{h} / u_{0}$ in connection with the empirical mode's frequency $f$. The mode pair IV with low frequency and high energy content corresponds to the motion of the vortex breakdown bubble. It will not be discussed in detail here as our research is concerned with the detection of helical coherent structures, i.e. mode pairs I and III shown in the figure. The SPOD results show that the mode pairs I (modes 31 and 32) and III (modes 11 and 12) account for $0.62 \%$ and $1.05 \%$ of the turbulent kinetic energy, and they are ranked 6th and 16th among all the extracted mode pairs by energy, respectively. Similar to the experimental results $\frac{43}{\text {, }}$ mode pair III has a larger energy content than mode pair I in this work. However, the energy content of these SPOD modes is smaller when compared to the PIV measurements, which can be attributed to a greater number of snapshots used in this study and the filtering effect from the PIV measurements. SPOD inherently assumes that the number of snapshots assembled can accurately express the modes in the flow field. A smaller SPOD database would reduce the flow dynamical 

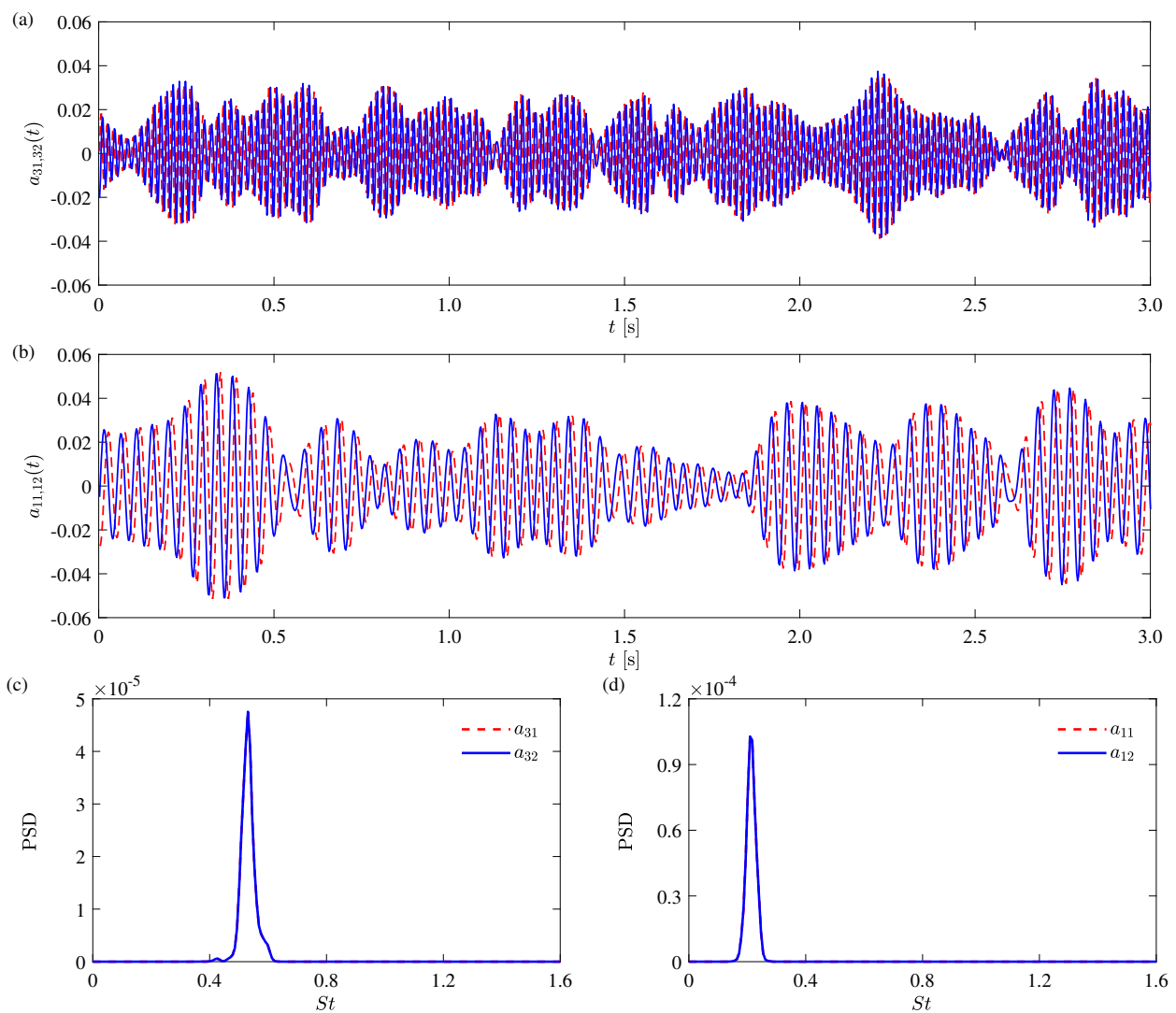

FIG. 11. Evolution of the temporal coefficients of mode pair I (a) and mode pair III (b). The power spectral density (PSD) of temporal coefficients from the energy-ranked mode pair I (c) and mode pair III $(d)$.

information, especially for the small scale modes. In addition, the limited PIV spatial resolution would make these small scale modes unrecognized in the experiment.

The evolution of the temporal coefficients of mode pair I and III are given respectively in figures 11(a) and 11(b). From the figures, we can see that the temporal coefficients in one mode pair almost have the same content except for a phase difference of $\pi / 2$, which verifies that the two harmonic modes can be interpreted as the orthogonal components of a precessing structure. The SPOD coefficients are the representative of flow dynamics. The evolution of the coefficients in magnitude demonstrates that these modes are not always present in the flow field, i.e. they are neutrally stable and have intermittent dynamics. Moreover, mode pair I is weaker than mode pair III as it has lower energy content and the maximum value of its temporal coefficients is smaller than that of mode pair III, which is in line with the experimental investigation both qualitatively and quantitatively 4 . The 
power spectral density of the corresponding linked mode temporal coefficients shown in figures 11(c) and 11(d) reveal that their spectra have a peak at Strouhal numbers 0.52 and 0.21, corresponding to frequencies of $55.24 \mathrm{~Hz}$ and $22.25 \mathrm{~Hz}$, respectively. The frequency of $22.25 \mathrm{~Hz}$ is confirmed by the spectra of velocity and pressure fluctuations at the stagnation point in figure 9 as well. Recently, the phase averaging of velocity fields in the turbulent annular swirling jet has shown a precessing helical structure at a St of 0.27 (a frequency of $28 \mathrm{~Hz}$ ) by Vanierschot et al. $\stackrel{42}{\text { }}$. This is also confirmed by the POD analysis of the pressure fields ${ }^{12}$ and SPOD analysis of the velocity fields ${ }^{43}$ in the same flow, where both a helical formation at $28 \mathrm{~Hz}$ and a double helical structure at $56 \mathrm{~Hz}$ were identified. The spatial structure of the modes at $x / D_{o}=0.9$ is shown in figure 12 . The spatial structure of the modes of pair I is shown in figures 12 (a) and 12 (b). The in-plane velocity vectors reveal 4 different vortex cores with a spatial angle of $\pi / 2$ between each core. Both spatial modes of the pair are shifted $\pi / 4$ in space, as is also seen in the study of Vanierschot et al. $\stackrel{43}{ }$. The spatial structure of the modes of pair III is shown in figures 12 (c) and 12 (d). The in-plane velocity vectors reveal 2 different vortex cores with a spatial angle of $\pi$ between each core. Both spatial modes of the pair are shifted $\pi / 2$ in space, as is also seen in the study of Vanierschot et al. $\stackrel{43}{.}$.

\section{Single and double helical structures}

The coherent structures can be identified by recombining the investigated mode pair with the time-averaged velocity field. Figures $13(\mathrm{a})$ and 13 (b) respectively present the vortical structures reconstructed by mode pairs I and III. It has a double helical formation in figure 13 (a) and a single helical formation in figure 13 (b). From figure 13 we can see that, the single vortical structure has larger size than the double one if the same threshold is selected, which means that the single-helix is more energetic than the double-helix. The single and double helical vortical structures found in this study both correspond to the spiral type of vortex breakdown. These spiral-shaped structures have high vorticity wrapped around the vortex breakdown bubble and rotate around the symmetry axis at a constant rate.

The phase portraits in figures 14(a) and 14(b) are circular in shape and they illustrate the harmonic relation of the SPOD modes in pairs I and III, respectively. POD analysis of the instantaneous three-dimensional pressure fields for this system by Percin et al. ${ }^{[2}$ gave 

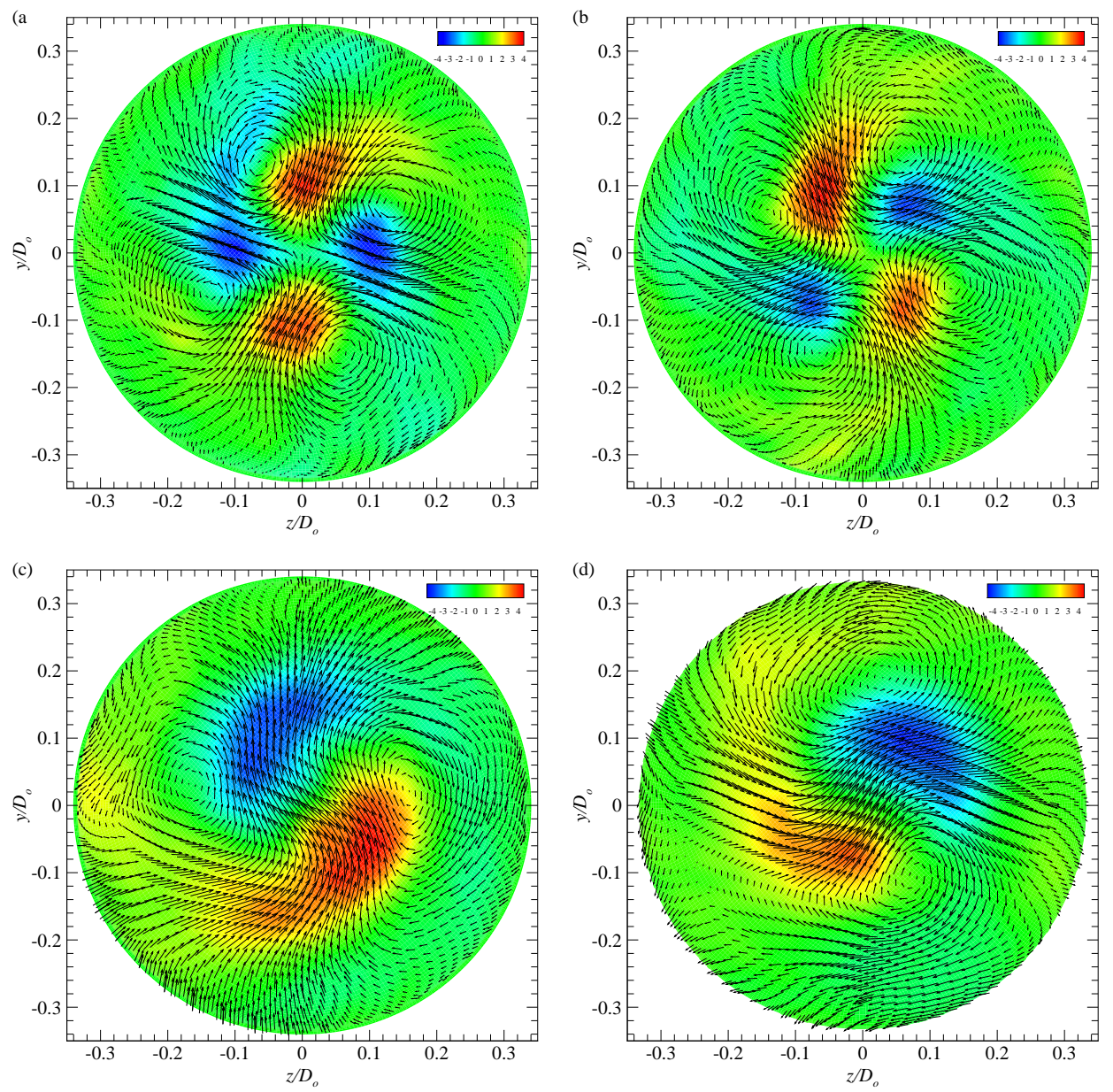

FIG. 12. The slices of SPOD modes at $x / D_{o}=0.9$ : (a) mode 31, (b) mode 32, (c) mode 11, and (d) mode 12. The colors in the figure shows the distribution of streamwise velocity (unit $\mathrm{m} / \mathrm{s}$ ), and the vectors indicate the radial and tangential velocity field.

both single and double helical structures, and the precessing frequency of the double helical structure was about two times larger than the single one. Hence, they suspected that the double helical formation is a second harmonic of the single helical one. However, in the present study, this is not the case. The relation between the temporal coefficient of mode pairs I and III shown in figure 14(c) is not shaped like an eight. In addition, the phase angles of these two mode pairs, i.e., $\phi_{\mathrm{I}}=\arctan \left(a_{31} / a_{32}\right)$ versus $\phi_{\mathrm{III}}=\arctan \left(a_{11} / a_{12}\right)$ given in figure $14(\mathrm{~d})$ does not present the circle dots arranged in diagonals. The connection between the single and double helical vortical structures identified here is in line with a later experimental study by Vanierschot et al. 13 . They showed that these structures are separate global models by global stability analysis. RANS simulations of the same flow case using a 


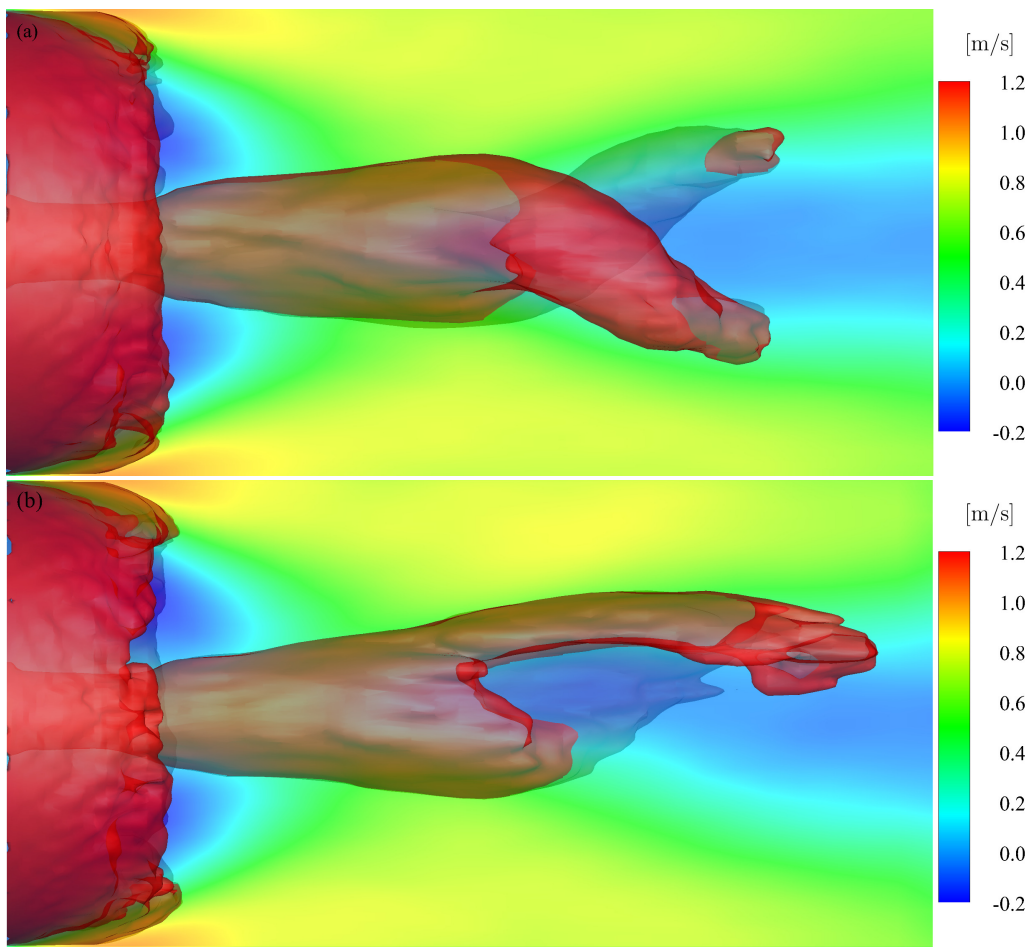

FIG. 13. The double (a) and single (b) helical structures identified by the isosurface of positive $Q=4 \times 10^{4} 1 / \mathrm{s}^{2}$. The slices show the contours of axial velocity in the $x-y$ plane.

RSM turbulence model shows that the latter predicts the double helix as a second harmonic of the single helix and hence LES can better capture the experimental observations ${ }^{63}$. Also, it is worth noting that the double helix structure is rarely observed in turbulent swirling jets and it is mainly reported in laminar swirling flow

To study the dynamics of the two large scale helical structures, the contribution of the coherent flow component to the production of the turbulent kinetic energy is calculated:

$$
P=-\left\langle\bar{u}_{i}^{\dagger} \bar{u}_{j}^{\dagger}\right\rangle \frac{\partial\left\langle\bar{u}_{i}\right\rangle}{\partial x_{j}} \text { with }\left\langle\bar{u}_{i}^{\dagger} \bar{u}_{j}^{\dagger}\right\rangle=\sum_{r}\left(\lambda_{p}^{r} \Phi_{p i}^{r} \Phi_{p j}^{r}+\lambda_{q}^{r} \Phi_{q i}^{r} \Phi_{q j}^{r}\right),
$$

where $\partial\left\langle\bar{u}_{i}\right\rangle / \partial x_{j}$ is the mean velocity gradient and $\left\langle\bar{u}_{i}^{\dagger} \bar{u}_{j}^{\dagger}\right\rangle$ are the second-order coherent stresses caused by the precessing motion. If $P$ is positive, then it means that energy is extracted from the mean motion to increase the kinetic energy of the coherent flow. Figures 15 (a) and 15(b) present the contours of the normal stresses of $\left\langle\bar{u}_{i}^{\dagger} \bar{u}_{j}^{\dagger}\right\rangle$ introduced respectively by the double and single helix. It is shown that the large coherent stresses for the double helical case lie in the region immediately downstream of the CRZ and in the shear layer between the vortex breakdown bubble and the jet, while the highest coherent stresses 

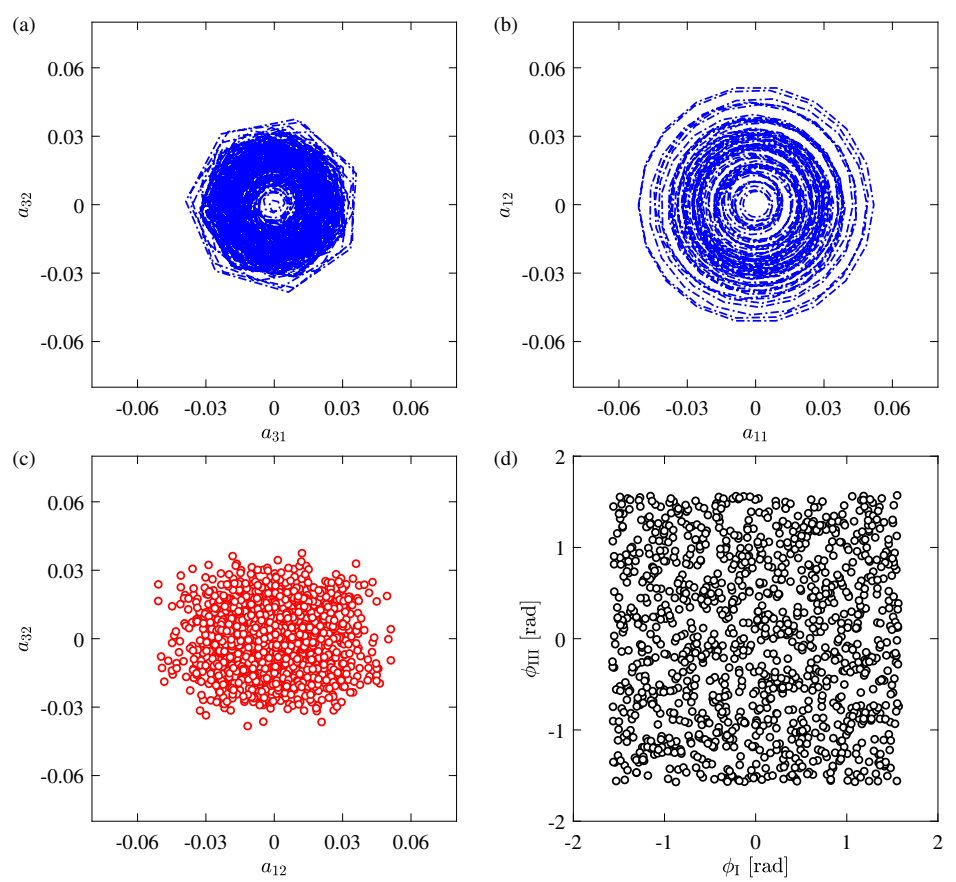

FIG. 14. Phase portrait of the mode temporal coefficients of mode pair I (a) and III (b). Phase portrait of the mode temporal coefficients between mode pair I and III (c) and their phase angles (d).

are close to the region where the central vortex core breaks up (around $x / D_{o} \approx 0.8$ ) for the single helical formation. The contours of $P$ calculated from mode pairs I and III are plotted in figures 16(a) and 16(b), respectively. The calculation of coherent production shows that the double helix extracts energy immediately downstream of the CRZ and in the shear layer between breakdown bubble and jet, and the single helix extracts energy from the shear layer between the vortex breakdown bubble and the jet. Regardless of the fact that the large values of coherent stresses and energy production move upstream due to the axial shift of the vortex breakdown bubble predicted by LES, the results agree with the experimental measurements in terms of their distribution and magnitude ${ }^{43}$.

\section{SUMMARY AND CONCLUSIONS}

In this paper, a turbulent annular swirling jet experiencing vortex breakdown with Reynolds number $R e=8500$ and swirl number $S w=0.38$ is simulated by means of LES using the localized dynamic $k$ model. The numerical data is validated by comparing the mean 

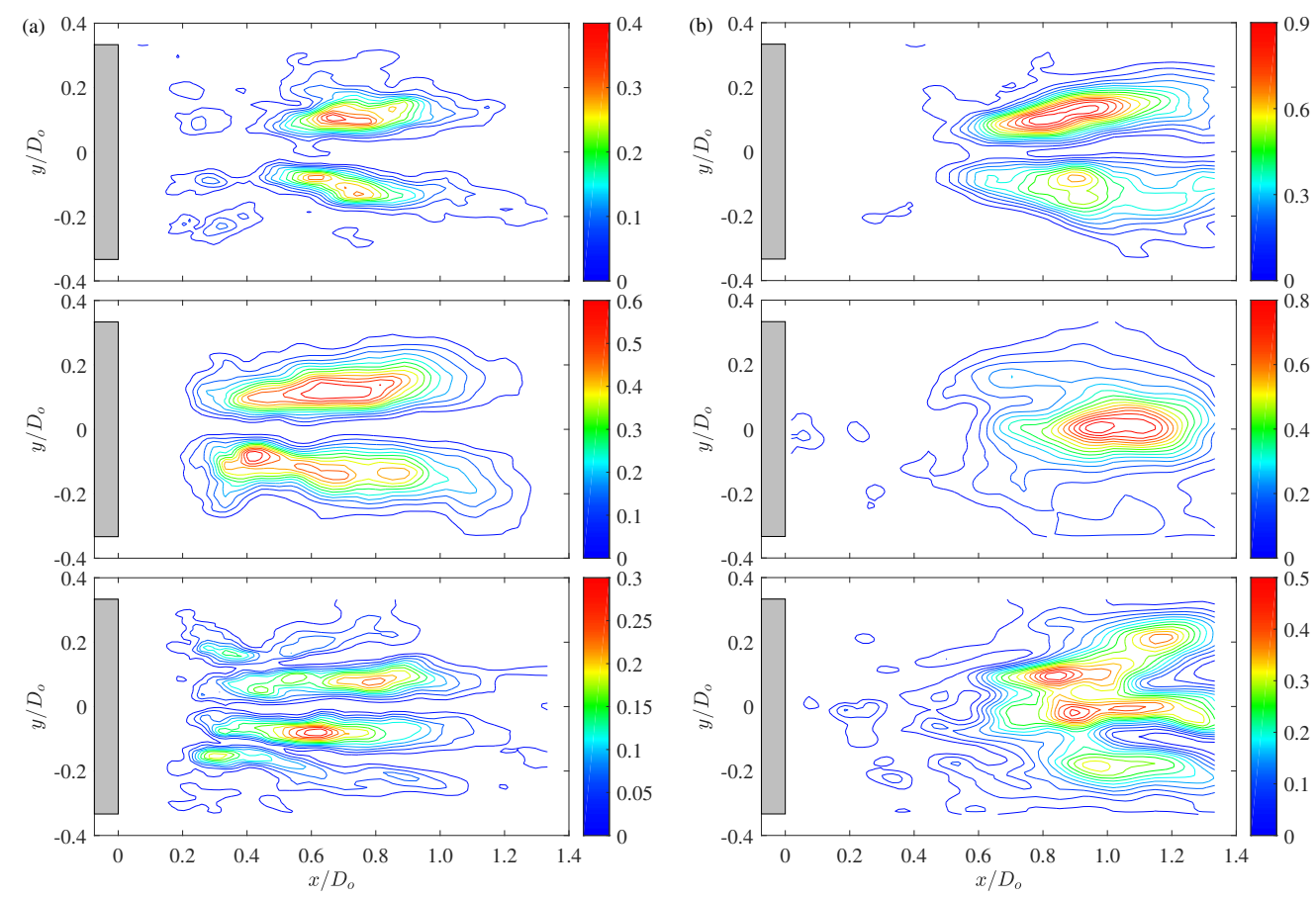

FIG. 15. Contours of the normal coherent stresses introduced by the double (a) and single (b) helical structure: $100 \times\left\langle\bar{u}^{\dagger} \bar{u}^{\dagger}\right\rangle / u_{0}^{2}, 100 \times\left\langle\bar{v}^{\dagger} \bar{v}^{\dagger}\right\rangle / u_{0}^{2}$, and $100 \times\left\langle\bar{w}^{\dagger} \bar{w}^{\dagger}\right\rangle / u_{0}^{2}$ from top to bottom.
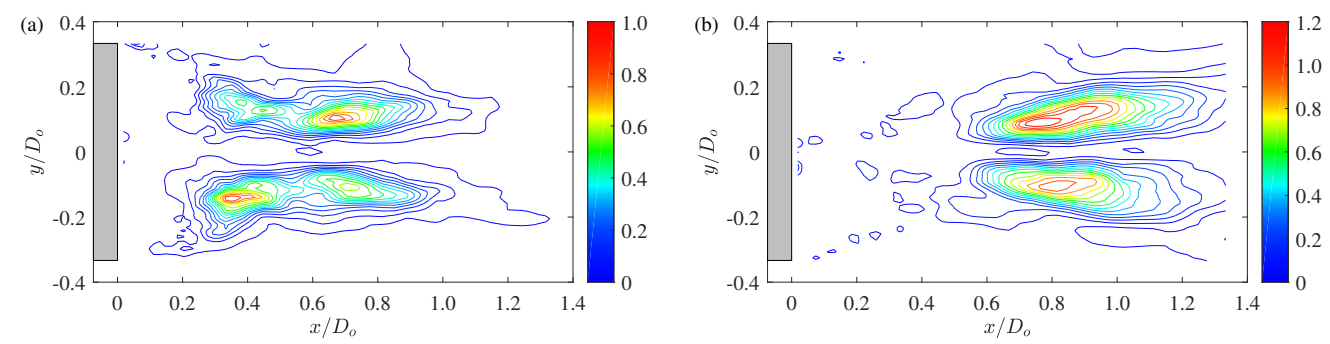

FIG. 16. Contours of the kinetic energy production of the double (a) and single (b) helical structure.

velocity and rms velocity fluctuation profiles with experimental measurements. Moreover, a precession frequency of $22 \mathrm{~Hz}$ of the precessing vortex core is found by the spectral analysis of the sampled velocity and pressure signals. Furthermore, spectral proper orthogonal decomposition is applied to detect the coherent structures in the flow field. It is found that, in addition to the single helical vortical structure usually reported in swirling flows, a double helical vortical structure is also identified. These single and double spiral type coherent structures with excessive vorticity are wrapped around the vortex breakdown bubble and rotate in the counter-swirl direction at Strouhal numbers 0.21 and 0.52 , respectively. The calculation of kinetic energy production contributed by these helical structures shows that 
the single helix mainly extracts energy from the shear layer between the vortex breakdown bubble and the jet, while the double helix extracts energy both immediately downstream of the CRZ and in the shear layer between breakdown bubble and jet.

\section{DATA AVAILABILITY STATEMENT}

The data that support the findings of this study are available from the corresponding author upon reasonable request.

\section{ACKNOWLEDGEMENTS}

Y. Zhang would like to acknowledge the support from the China Scholarship Council (Grant No. 201706890016). The simulations were carried out on the Tier-2 cluster of the Flemish Supercomputer Center (Vlaams Supercomputer Centrum - VSC).

\section{REFERENCES}

${ }^{1}$ M. Karami, L. Carassale, and H. Hangan, "Statistical and modal analysis of surface pressure fluctuations in tornado-like vortices," Physics of Fluids 32, 075109 (2020).

${ }^{2}$ M. Karami, H. Hangan, L. Carassale, and H. Peerhossaini, "Coherent structures in tornado-like vortices," Physics of Fluids 31, 085118 (2019).

${ }^{3}$ A. K. Gupta, D. G. Lilley, and N. Syred, Swirl flows (Tunbridge Wells, Kent, England, Abacus Press, 1984).

${ }^{4}$ J. Beér and N. Chigier, Combustion Aerodynamics (Krieger Pub Co, 1983).

${ }^{5}$ S. Candel, D. Durox, T. Schuller, J.-F. Bourgouin, and J. P. Moeck, "Dynamics of swirling flames," Annual Review of Fluid Mechanics 46, 147-173 (2014).

${ }^{6}$ B. Zhang, M. Shahsavari, Z. Rao, S. Yang, and B. Wang, "Contributions of hydrodynamic features of a swirling flow to thermoacoustic instabilities in a lean premixed swirl stabilized combustor," Physics of Fluids 31, 075106 (2019).

${ }^{7}$ N. Jalalatian, S. Tabejamaat, B. Kashir, and M. EidiAttarZadeh, "An experimental study on the effect of swirl number on pollutant formation in propane bluff-body stabilized swirl diffusion flames," Physics of Fluids 31, 055105 (2019). 
${ }^{8}$ J. Fröhlich, M. García-Villalba, and W. Rodi, "Scalar mixing and large-scale coherent structures in a turbulent swirling jet," Flow, Turbulence and Combustion 80, 47-59 (2008).

${ }^{9}$ M. Vanierschot, T. Persoons, and E. Van den Bulck, "A new method for annular jet control based on cross-flow injection," Physics of Fluids 21, 025103 (2009).

${ }^{10}$ A. V. Brito Lopes, N. Emekwuru, B. Bonello, and E. Abtahizadeh, "On the highly swirling flow through a confined bluff-body," Physics of Fluids 32, 055105 (2020).

${ }^{11} \mathrm{M}$. Vanierschot and E. Van den Bulck, "Influence of swirl on the initial merging zone of a turbulent annular jet," Physics of Fluids 20, 105104 (2008).

${ }^{12}$ M. Percin, M. Vanierschot, and B. van Oudheusden, "Analysis of the pressure fields in a swirling annular jet flow," Experiments in Fluids 58, 166 (2017).

${ }^{13}$ D. Peckham and S. Atkinson, Preliminary results of low speed wind tunnel tests on a gothic wing of aspect ratio 1.0 (Royal Aircraft Establishment, 1957).

${ }^{14} \mathrm{~N}$. Syred, "A review of oscillation mechanisms and the role of the precessing vortex core (PVC) in swirl combustion systems," Progress in Energy and Combustion Science 32, 93-161 (2006).

${ }^{15}$ M. Hall, "Vortex breakdown," Annual Review of Fluid Mechanics 4, 195-218 (1972).

${ }^{16}$ M. Escudier, "Vortex breakdown: observations and explanations," Progress in Aerospace Sciences 25, 189-229 (1988).

${ }^{17}$ I. Naumov, B. Sharifullin, M. Tsoy, and V. Shtern, "Dual vortex breakdown in a two-fluid confined flow," Physics of Fluids 32, 061706 (2020).

${ }^{18}$ L. Carrion, I. Naumov, B. Sharifullin, M. Herrada, and V. Shtern, "Formation of dual vortex breakdown in a two-fluid confined flow," Physics of Fluids 32, 104107 (2020).

${ }^{19} \mathrm{R}$. Sujith and V. Unni, "Complex system approach to investigate and mitigate thermoacoustic instability in turbulent combustors," Physics of Fluids 32, 061401 (2020).

${ }^{20}$ O. Lucca-Negro and T. O'Doherty, "Vortex breakdown: a review," Progress in Energy and Combustion Science 27, 431-481 (2001).

${ }^{21}$ C. Cala, E. Fernandes, M. Heitor, and S. Shtork, "Coherent structures in unsteady swirling jet flow," Experiments in Fluids 40, 267-276 (2006).

${ }^{22}$ G. Berkooz, P. Holmes, and J. L. Lumley, "The proper orthogonal decomposition in the analysis of turbulent flows," Annual Review of Fluid Mechanics 25, 539-575 (1993).

${ }^{23}$ P. J. Schmid, "Dynamic mode decomposition of numerical and experimental data," Journal of Fluid Mechanics 656, 5-28 (2010). 
${ }^{24}$ M. Sieber, C. O. Paschereit, and K. Oberleithner, "Spectral proper orthogonal decomposition," Journal of Fluid Mechanics 792, 798-828 (2016).

${ }^{25} \mathrm{M}$. Vanierschot and G. Ogus, "Experimental investigation of the precessing vortex core in annular swirling jet flows in the transitional regime," Experimental Thermal and Fluid Science 106, 148-158 (2019).

${ }^{26}$ P. A. Kadu, Y. Sakai, Y. Ito, K. Iwano, M. Sugino, T. Katagiri, T. Hayase, and K. Nagata, "Application of spectral proper orthogonal decomposition to velocity and passive scalar fields in a swirling coaxial jet," Physics of Fluids 32, 015106 (2020).

${ }^{27}$ S. McIlwain and A. Pollard, "Large eddy simulation of the effects of mild swirl on the near field of a round free jet," Physics of Fluids 14, 653-661 (2002).

${ }^{28}$ X. Lu, S. Wang, H.-G. Sung, S.-Y. Hsieh, and V. Yang, "Large-eddy simulations of turbulent swirling flows injected into a dump chamber," Journal of Fluid Mechanics 527, 171-195 (2005).

${ }^{29}$ M. García-Villalba, J. Fröhlich, and W. Rodi, "Identification and analysis of coherent structures in the near field of a turbulent unconfined annular swirling jet using large eddy simulation," Physics of Fluids 18, 055103 (2006).

${ }^{30} \mathrm{M}$. García-Villalba and J. Fröhlich, "LES of a free annular swirling jet-dependence of coherent structures on a pilot jet and the level of swirl," International Journal of Heat and Fluid Flow 27, 911-923 (2006).

${ }^{31}$ M. García-Villalba, J. Fröhlich, and W. Rodi, "Numerical simulations of isothermal flow in a swirl burner," Journal of Engineering for Gas Turbines and Power 129, 377-386 (2007).

${ }^{32}$ W. Malalasekera, K. Ranga Dinesh, S. Ibrahim, and M. Kirkpatrick, "Large eddy simulation of isothermal turbulent swirling jets," Combustion Science and Technology 179, 1481-1525 (2007).

${ }^{33}$ S. Wang, V. Yang, G. Hsiao, S.-Y. Hsieh, and H. C. Mongia, "Large-eddy simulations of gas-turbine swirl injector flow dynamics," Journal of Fluid Mechanics 583, 99-122 (2007).

${ }^{34}$ K. R. Dinesh and M. Kirkpatrick, "Study of jet precession, recirculation and vortex breakdown in turbulent swirling jets using LES," Computers \& Fluids 38, 1232-1242 (2009).

${ }^{35}$ Y. Yang and S. K. Kær, "Large-eddy simulations of the non-reactive flow in the Sydney swirl burner," International Journal of Heat and Fluid Flow 36, 47-57 (2012).

${ }^{36}$ K. R. Dinesh, M. Kirkpatrick, and K. W. Jenkins, "Investigation of the influence of swirl on a confined coannular swirl jet," Computers \& Fluids 39, 756-767 (2010). 
${ }^{37}$ W. Jones, S. Lyra, and S. Navarro-Martinez, "Large eddy simulation of turbulent confined highly swirling annular flows," Flow, Turbulence and Combustion 89, 361-384 (2012).

${ }^{38}$ H. Zhang, C. Han, T. Ye, J. Zhang, and Y. Chen, "Large eddy simulation of unconfined turbulent swirling flow," Science China Technological Sciences 58, 1731-1744 (2015).

${ }^{39}$ B. Wegner, A. Maltsev, C. Schneider, A. Sadiki, A. Dreizler, and J. Janicka, "Assessment of unsteady RANS in predicting swirl flow instability based on les and experiments," International Journal of Heat and Fluid Flow 25, 528-536 (2004).

${ }^{40}$ D. Dunham, A. Spencer, J. J. McGuirk, and M. Dianat, "Comparison of unsteady Reynolds averaged Navier-Stokes and large eddy simulation computational fluid dynamics methodologies for air swirl fuel injectors," Journal of Engineering for Gas Turbines and Power 131, 011502 (2009).

${ }^{41}$ S. Taamallah, Y. Dagan, N. Chakroun, S. J. Shanbhogue, K. Vogiatzaki, and A. F. Ghoniem, "Helical vortex core dynamics and flame interaction in turbulent premixed swirl combustion: A combined experimental and large eddy simulation investigation," Physics of Fluids 31, 025108 (2019).

${ }^{42}$ M. Vanierschot, M. Percin, and B. van Oudheusden, "Double helix vortex breakdown in a turbulent swirling annular jet flow," Physical Review Fluids 3, 034703 (2018).

${ }^{43}$ M. Vanierschot, J. S. Müller, M. Sieber, M. Percin, B. W. van Oudheusden, and K. Oberleithner, "Single- and double-helix vortex breakdown as two dominant global modes in turbulent swirling jet flow," Journal of Fluid Mechanics 883 (2020).

${ }^{44}$ W.-W. Kim and S. Menon, "A new dynamic one-equation subgrid-scale model for large eddy simulations," in 33rd Aerospace Sciences Meeting and Exhibit (1995) p. 356.

${ }^{45}$ W.-W. Kim and S. Menon, "An unsteady incompressible Navier-Stokes solver for large eddy simulation of turbulent flows," International Journal for Numerical Methods in Fluids 31, 983-1017 (1999).

${ }^{46}$ E. R. Van Driest, "On turbulent flow near a wall," Journal of the Aeronautical Sciences 23, 1007-1011 (1956).

${ }^{47}$ P. Moin and J. Kim, "Numerical investigation of turbulent channel flow," Journal of Fluid Mechanics 118, 341-377 (1982).

${ }^{48}$ J. Smagorinsky, "General circulation experiments with the primitive equations: I. the basic experiment," Monthly Weather Review 91, 99-164 (1963). 
${ }^{49}$ M. Germano, U. Piomelli, P. Moin, and W. H. Cabot, "A dynamic subgrid-scale eddy viscosity model," Physics of Fluids A: Fluid Dynamics 3, 1760-1765 (1991).

${ }^{50}$ D. K. Lilly, "A proposed modification of the germano subgrid-scale closure method," Physics of Fluids A: Fluid Dynamics 4, 633-635 (1992).

${ }^{51}$ A. Lopes, N. Emekwuru, B. Bonello, and E. Abtahizadeh, "On the highly swirling flow through a confined bluff-body," Physics of Fluids 32, 055105 (2020).

${ }^{52} \mathrm{~W}$. Leuckel, Swirl intensities, swirl types and energy losses of different swirl generating devices (International Flame Research Foundation, Ijmuiden, 1967).

${ }^{53}$ T. udihara, L. Goldstein Jr, and M. Mori, "The three-dimensional numerical aerodynamics of a movable block burner," Brazilian Journal of Chemical Engineering 20, 391-401 (2003).

${ }^{54}$ https://www. openfoam.com/.

${ }^{55}$ J. H. Ferziger, M. Perić, and R. L. Street, Computational methods for fluid dynamics, Vol. 3 (Springer, 2002).

${ }^{56}$ S. B. Pope, "Ten questions concerning the large-eddy simulation of turbulent flows," New journal of Physics 6, 35 (2004).

${ }^{57}$ A. K. M. F. Hussain and W. C. Reynolds, "The mechanics of an organized wave in turbulent shear flow," Journal of Fluid Mechanics 41, 241-258 (1970).

${ }^{58} \mathrm{Z}$. Xia, X. Han, and J. Mao, "Assessment and validation of very-large-eddy simulation turbulence modeling for strongly swirling turbulent flow," AIAA journal 58, 148-163 (2020). ${ }^{59}$ A. De Santis, A. Clements, A. Pranzitelli, D. Ingham, and M. Pourkashanian, "Assessment of the impact of subgrid-scale stress models and mesh resolution on the les of a partiallypremixed swirling flame," Fuel 281, 118620 (2020).

${ }^{60}$ S. V. Alekseenko, A. V. Bilsky, V. M. Dulin, and D. M. Markovich, "Experimental study of an impinging jet with different swirl rates," International Journal of Heat and Fluid Flow 28, 1340-1359 (2007).

${ }^{61} \mathrm{H}$. Sheen, W. Chen, and S. Jeng, "Recirculation zones of unconfined and confined annular swirling jets," AIAA Journal 34, 572-579 (1996).

${ }^{62}$ J. Jeong and F. Hussain, "On the identification of a vortex," Journal of Fluid Mechanics 285, 69-94 (1995).

${ }^{63}$ Y. Zhang and M. Vanierschot, "Modeling capabilities of unsteady rans for the simulation of turbulent swirling flow in an annular bluff-body combustor geometry," Applied Mathematical Modelling 89, 1140-1154 (2021). 
${ }^{64} \mathrm{H}$. Liang and T. Maxworthy, "An experimental investigation of swirling jets," Journal of Fluid Mechanics 525, 115-159 (2005).

${ }^{65}$ P. Meliga, F. Gallaire, and J.-M. Chomaz, "A weakly nonlinear mechanism for mode selection in swirling jets," Journal of Fluid Mechanics 699, 216-262 (2012). 